\title{
SARS-CoV-2 gained the unique spike protein S1-N- Terminal Domain from the promising pangolins mixing vessel: A novel clue for the pandemic outbreak
}

Perumal desingu ( $\square$ padesingu@gmail.com )

padesingu@gmail.com

K Nagarajan

Letter

Keywords:

Posted Date: July 16th, 2021

DOl: https://doi.org/10.21203/rs.3.rs-721043/v1

License: (c) (1) This work is licensed under a Creative Commons Attribution 4.0 International License.

Read Full License 
1 SARS-CoV-2 gained the unique spike protein S1-N-Terminal Domain from the 2 promising pangolins mixing vessel: A novel clue for the pandemic outbreak

3

4 Perumal Arumugam Desingu ${ }^{1 \#}$, K. Nagarajan ${ }^{2}$

$5{ }^{1}$ Department of Microbiology and Cell Biology, Indian Institute of Science, Bengaluru, India

$6{ }^{2}$ Department of Veterinary Pathology, Madras Veterinary College, Vepery, Chennai, 600007,

7 Tamil Nadu Veterinary and Animal Sciences University (TANUVAS),

8

9

10

Keywords: SARS-CoV-2, the origin of SARS-CoV-2, spike protein, S1-N-Terminal

Domain, pandemic outbreak, pangolin mixing vessels

13

Running title: SARS-CoV-2 gained the novel S1-NTD

\# Corresponding authors,

Perumal Arumugam Desingu, Department of Microbiology and Cell Biology, Division of Biological Sciences, Indian Institute of Science, Bangalore - 560012, India. Email: perumald@iisc.ac.in; padesingu@gmail.com Phone: +91 802293 2068, Fax: +91 802360 2697 
The clue behind the emergence of SARS-CoV-2 remains a huge debate among the virologist, policymakers, and general public, while the gain-of-function hypothesizes mostly focused on the furin cleavage site and receptor-binding domain (RBD) of the spike (S) protein. Here, we report that the SARS-CoV-2 gained the novel human-specific spike protein S1-N-Terminal Domain (S1-NTD) (Type-I) which is present only in SARS-CoV-2. Interestingly, SARSCoV-2-rB-CoV showed unique bat-specific Type-II and Type-III-S1-NTD, which is not present in other SARS coronaviruses, including SARS-CoV-2 variants. We also found widespread recombination and selection pressure in the S1-NTDs of the bat viruses. In addition, the Pangolin/GX/2018 and Pangolin/Guangdong/2019 lineages showed close identity (73-79\%) with the Type-I-S1-NTD and Type-II-S1-NTD respectively. This justifies that the pangolin is the mixing vessel (intermediate host) to exchange the bat-specific TypeII-S1-NTD in the SARS-CoV-2-rB-CoV into Type-I-like-S1-NTD in pangolin through recombination. Furthermore, the pangolin virus with Type-I-like-S1-NTD jumped into humans which then transformed into SARS-CoV-2 with Type-I-S1-NTD by host selection pressure. Remarkably, we characterized the bat/Cambodia virus as a recombinant SARSCoV-2/RatG13 with the S1-NTD of bat-SARS-like viruses; while only bat/RatG13 with Type-I-S1-NTD established a huge pandemic outbreak. Additionally, recent SARS-CoV-2 S1-NTD specific neutralizing antibody-based studies support the role of S1-NTD in the postattachment of the virus; fusion, virus dissemination, and cell-cell fusion thereby prevent the onset of infection; and most of the SARS-CoV-2 variants with increased transmissibility were linked to the S1-NTD mutations. Collectively, our results strongly suggest that the gain of Type-I-S1-NTD in the SARS-CoV-2 is the reason for the pandemic outbreak.

\section{Introduction}

The sudden emergence of highly adaptable and transmissible SARS-CoV-2 in the human population in December 2019, in Wuhan, China ${ }^{1,2}$, raised the question about its origin. However, pangolins were considered an intermediate host between humans and $b^{2} t^{2-5}$, due to the occurrence of almost identical spike protein receptor-binding domain (S1-RBD) in pangolin-CoVs and SARS-CoV-2 $2^{5-7}$. However, all SARS-CoV-2 related bat and pangolin viruses except RatG13 showed much lower sequence identity in the spike gene ${ }^{3-5,8}$, which is reported to be critical in determining the virus entry and tissue tropism, host-range, humanhuman infection, and host immune responses ${ }^{9-15}$. The SARS-CoV-2 spike protein is divided into a signal peptide, $\mathrm{S} 1$, and $\mathrm{S} 2$ subunits. The $\mathrm{S} 1$ subunit is further divided into two independent receptor binding domains such as sugar receptor binding S1-NTD (14-305 amino acid residues) and protein receptor binding S1-RBD (319-541 amino acid residues) $)^{10,14,16,17}$. Further, it is widely accepted that the coronaviruses obtained the S1-NTD from host galectins through gene capture, and then gained the S1-RBD through gene duplication from the S1-NTD ${ }^{13,14}$. Due to the presence of the S1-RBD in the tip of the spike head whereas S1-NTD is perhaps positioned underneath the S1-RBD ${ }^{14,18,19}$; S1-RBD has more likelihood to expose to the host immune system and evolve at a faster rate than S1$\mathrm{NTD}^{14}$. Further, the presence of extensive N-linked glycan shielding in the S1-NTD is expected to play a role in the immune evasion ${ }^{9,20-22}$. Therefore, the less diversified S1-NTDs 
are considered a more reliable domain for the coronaviruses to bind the sugar receptors thereby facilitating the S1-RBDs to search their high-affinity protein receptors ${ }^{14}$. Remarkably, the level of diversity and interaction of these domains with the host receptor is a critical determinant of the coronavirus host range, cross-species infection, tissue tropism, and host immune responses ${ }^{13-15}$. However, the genetic diversity and evolutionary origin of the S1RBD were highly characterized in the SARS-CoV-2 ${ }^{3-5,16,23,24}$. On the other hand, the genetic diversity and evolutionary origin of the SARS-CoV-2 S1-NTDs are not yet fully established.

We present evidence for the emergence of the SARS-COV-2 pandemic outbreak due to the gain of novel Type-I-S1-NTD in its spike protein. Further, we report the existence of batspecific different types of S1-NTD in the SARS-COV-2-rB-CoVs and report the different recombinant SARS-COV-2-rB-CoVs especially in the regions of S1-NTD and S1-RBD. Finally, we report the evidence to suggest pangolins as a mixing vessel to generate the human-specific Type-I-S1-NTDs from bat-specific Type-II-S1-NTDs.

\section{Genetic diversity in SARS-CoV-2 related bat coronavirus}

Our analysis revealed the presence of two clades of SARS-CoV-2 related bat coronavirus (SARS-CoV-2-rB-CoV) at the complete genome level (Fig. 1a). The Clade-I viruses (RaTG13; RShSTT182h, RmYN02; RacCS203 andRpYN06) exposed the nucleotide diversity between $4-10 \%$ with SARS-CoV-2 and within the clade-I viruses. The clade II viruses (bat/PrC31, bat-SL-CoVZC45, and bat-SL-CoVZXC21) have a diversity of 10-13\% with SARS-CoV-2 and clade-I viruses (Extended Data Fig. 1a). The Pangolin/Guangdong/2019 lineage showed a close relationship with the clade-II SARS-CoV2-rB-CoV (Extended Data Fig. 1a \& 1b). Remarkably, Pangolin/GX/2018 lineage possessed evolutionary intermediate genetic diversity between SARS-CoV-1 and SARSCoV-2 (Extended Data Fig. 1a \& 1b). Next, at the Spike complete gene level, we observed genetic diversity of $25-35 \%$ among the SARS-CoV-2-rB-CoV. However, genetic diversity between the SARS-CoV-2-rB-CoV and SARS-CoV-1-rB-CoV (SARS-CoV-1 related-bat$\mathrm{CoVs}$ ) is in the range of 20-26\% (Extended Data Fig. 1c \& 1d). These results indicate that the genetic diversity in the $\mathrm{S}$ gene is a random diversity without specific to certain lineage, perhaps due to the genetic recombination events in this gene.

\section{Genomic region's specific genetic diversity in SARS-CoV-2}

Next, we observed that the SARS-CoV-2 displayed four hypervariable regions in comparison to SARS-CoV-1 and one hyper-variable region in comparison to SL-CoVZXC21 and bat-SLCoVZC45 in SimPlot analysis (Fig. 1b). However, we are unable to detect any significant recombination between SARS-CoV-2 and SARS-CoV-1 in BootScan method of analysis (Extended Data Fig. 1e). Further, in our NCBI BLAST analysis, clade-II SARS-CoV-2-rB$\mathrm{CoV}$ and SARS-CoV-1 showed different region's specific high and low nucleotide sequence diversities compared to Wuhan-Hu-1 (Extended Data Fig. 1f); owing to the widespread recombination events in this virus ${ }^{1,6,25,26}$. According to our SimPlot and NCBI BLAST analysis, we divided the SARS-CoV-2 genome sequences into 11 different regions. The genomic portions of regions $1,2,4,5,8,10$, and 11 displayed low genetic diversity among 
the SARS-CoV-2, SARS-CoV-2-rB-CoV, and SARS-CoVs (Extended Data Fig. 2a-d; Extended Data Fig. 2g-h; Extended Data Fig. 3a-b; Extended Data Fig. 3e-f; Extended Data Fig. 4a-d).

On the other hand, regions with substantial genetic diversity, such as region 3 and region 9 (protein 8 ) revealed 3-10\% genetic diversity among SARS-CoV-2 and SARS-CoV-2-rB-CoV (clade-I and clade-II), and over $40 \%$ genetic diversity against SARS-CoV-1-rB-CoVs (Extended Data Fig. 2e-f; Extended Data Fig. 3g-h). This indicates that these regions are the novel signature sequences of SARS-CoV-2 and SARS-COV-2-rB-CoVs. Further, the high diversity portion of region 7 (which belongs to the S1-RBD) on the other hand, has revealed two distinct clusters (Extended Data Fig. 3c-d). The SARS-CoV-2, RaTG13, RShSTT182, RShSTT200, Pangolin/GX/2018, and Pangolin/Guangdong/2019 formed a cluster-I. Surprisingly, the SARS-CoV-2-rB-CoVs of bat-SL-CoVZXC21, bat-SL-CoVZC45, RpYN06, bat/PrC31, bat/RacCS203, RmYN02 formed cluster-II with SARS-CoV-1-rB-CoV. This indicates the widespread recombination in the region.

Surprisingly, the most diversified section of region 6 (which contains the S1-NTD) had a maximum diversity of $90 \%$ among the viruses used in this study (Extended Data Fig. 5a-b). The SARS-CoV-2 and SARS-COV-2-rB-CoVs established three distinct clades (Type-I to III-S1-NTD) in this region (Fig. 1c-e, Supplementary Fig. 1a-b). The SARS-CoV-2 and RaTG13 formed Type-I-S1-NTD with intra-type nucleotide diversity of 6\% (1.5\% amino acid (aa) diversity) and inter-type variability of $44-85 \%$ (37-92\% aa diversity) (Fig. 1d; Extended Data Fig. 5a). Next, Pangolin/GX/2018 formed Type-I-like-S1-NTD and intertype variability of 27-90\% (27-28\% diversity with Type-I-S1-NTD) (Fig. 1d; Extended Data Fig. 5a). Similarly, Type-II-S1-NTD was established by SL-CoVZXC21, bat-SLCoVZC45, RpYN06, and Bat/PrC31, with intra-type diversity of 2-25\% (1-16\% aa diversity) and $44-83 \%$ (40-92\% aa diversity) inter-type variability (Fig. 1d; Extended Data Fig. 5a). Conversely, Type-III-S1-NTD was generated by RmYN02 and Bat coronavirus RacCS203, with intra-type diversity of 6\% and inter-type diversity of 68-91\% (Fig. 1d; Extended Data Fig. 5a). Interestingly, SARS-CoV-2-rB-CoVs of RShSTT200 and RShSTT182 revealed significant genetic diversity of $69-75 \%$ with all these three types (SARS-CoV-2 and SARSCoV-2-rB-CoVs) of S1-NTDs but had a tighter link with S1-NTDs of highly varied SARSCoV-1-rB-CoVs (Fig. 1d; Extended Data Fig. 5a). According to our findings, none of the human viral isolates (SARS-CoV-2 variants/lineages) formed an evolutionary intermediate between SARS-CoV-2 and bat/RatG13 in this region (Supplementary Fig1a-b), which indicates that all variants/lineages of SARS-CoV-2 originated from the base of NC_045512.2/Wuhan-Hu-1 Type-I-S1-NTD.

Moreover, Pangolin/GX/2018 lineage is the closest in this region to SARS-CoV-2 through Type-I-like-S1-NTD with 27\% and 12\% nucleotide and amino acid diversity, respectively (Fig. 1d; Extended Data Fig. 5a). But, Pangolin/Guangdong/2019 lineage is there in this region with $21-24 \%$ and $15 \%$, nucleotide, and amino acid diversity respectively, with TypeII-S1-NTDs of SARS-CoV-2-rB-CoVs (Fig. 1d; Extended Data Fig. 5a). These findings imply that only Type-I-S1-NTD (region 6) is found in the human population in the ongoing pandemic outbreak, whereas Type-II and III-S1-NTD were found only in the bat population. 
Pangolin virus lineages contain both SARS-CoV-2 human-like (Type-I-like-S1-NTD) and bat-like (TypeII-like-S1-NTD) viruses, indicating that pangolins could be used as a mixing vessel (intermediate host) for human and bat viruses to generate human-specific Type-I-S1NTD which represent the ongoing outbreak.

\section{Recombinant events in the SARS-CoV-2-rB-CoVs}

In our recombination analysis, we confirmed that the bat/Cambodia/RShSTT200 and bat/Cambodia/RShSTT182 are the recombinant SARS-CoV-2 with S1-NTD of SARS-like bat coronavirus (Fig.2a-b; Extended Data Fig. 6a-b; Supplementary Fig. 2a-b). Similarly, our analysis also confirmed the bat/China/RpYN06 which has unique Type-II-S1-NTD is a recombinant SARS-CoV-2 with S1-RBD of SARS-like bat coronaviruses (Extended Data Fig. 6c-d; Supplementary Fig. 2c). Next, we observed that the Bat/RacCS203 (which displays Type-III-S1-NTD) is a recombinant SARS-COV-2 with S1-RBD of SARS-like bat coronaviruses (Extended Data Fig. 6e-f; Supplementary Fig. 2d). Further, we characterized the Bat/Yunnan/RmYN02 (which exhibits Type-III-S1-NTD) as a recombinant SARS-CoV-2 with S1-RBD of SARS-like bat coronaviruses; further, it holds unique protein-8 (Extended Data Fig. 6g-h; Supplementary Fig. 2e). Interestingly, the clade-II of SARS-CoV-2-rBCoVs at the complete genome level which includes bat coronavirus isolate PrC31/China/2018, bat-SL-CoVZC45, bat-SL-CoVZXC21(which exhibit Type-II-S1-NTD) are the recombinant SARS-CoV-2 with some part of pp1ab and S1-RBD of SARS-like bat coronaviruses (Fig. 2c; Extended Data Fig. 7a-e; Supplementary Fig. 2f-h). More importantly, Pangolin/Guangdong/2019 lineage which has high sequence identity with SARS-CoV-2 S1-RBD, were displayed Type-II-S1-NTD in its genome, while Pangolin/GX/2018 lineage displayed the Type-I-like-S1-NTD and SARS-CoV-2-like-S1RBD (Fig 1d; Fig. 2d; Extended Data Fig. 3c-d; Extended Data Fig. 5a). Collectively, these results indicate the presence of widespread recombination in the SARS-COV-2-rBCoVs especially in the regions of S1-NTD and S1-RBD.

\section{Selection pressure in the S1-NTDs}

The S1-NTDs nucleotide sequence-based effective number of codon usage (ENc) suggest that the SARS-CoV-2 and bat-SARS viruses have moderate and minor codon use bias respectively (Fig 3a). Following that, in ENc-GC3s plot analysis ${ }^{27,28}$, we observed that a vast majority of the points fall below the expected curve in the ENc-GC3s plot analysis for the S1NTD (region 6) of SARS-CoV-2, SARS-CoV-1, pangolin SARS coronavirus, and bat SARS viruses (Fig 3b), indicating the strong presence of selection pressure, rather than mutation pressure. Next, our neutrality plot analysis revealed that the S1-NTD (region 6) of SARSCoV-2 had a slope of $0.09669(\mathrm{Y}=0.09669 * \mathrm{X}+37.28, \mathrm{R} 2=0.04538 ; \mathrm{p}=0.0093)$ (Fig 3c), indicating that the mutation pressure and natural selections were $9.7 \%$ and $90.3 \%$, respectively. Similarly, we observed the substantial selection pressure, rather than mutation pressure, in the S1-NTD (region 6) of SARS-Cov-2-rBat-CoVs, Pangolin-CoVs, SARS coronavirus, and bat SARS viruses (Extended Data Fig. 8a-g). We also ran a Parity rule 2 bias study and found that the uneven T3>A3 and C3>G3 numbers in the S1-NTD (region 6) of SARS-Cov-2 and SARS-Cov-2-rBat sequences (Fig. 3d) suggesting the existence of 
mutation and selection pressure. Taken altogether, these results indicate that the presence of strong selection pressure in this region which shaping the virus evolution.

\section{Discussions}

In this work, we report that the SARS-CoV-2 gained the novel S1-NTD in its genome. Most of the studies focused on the rapid spread of the SARS-CoV-2 due to its S1-RBD diversity to binds with the human ACE2 receptor and the presence of a specific furin cleavage site $(\mathrm{FCS})^{4,29-35}$. However, the furin cleavage site is not only present in SARS-CoV-2, but also present in MERS-CoV, HKU1, and HCoV-OC43 ${ }^{4,32,34,36}$, and the SARS-CoV-2 without FCS also can infect target cells efficiently in the presence of trypsin or human airway trypsin-like protease $(\mathrm{HAT})^{37}$. Further, the S1-RBD for the human ACE2 is also present in SARS-CoV-1; however, SARS-CoV-2 has improved binding to ACE2 receptor than SARS-CoV-1 ${ }^{38,39}$. Moreover, SARS-CoV-2 has been shown to infect efficiently 293T and Vero cells without human ACE2 transfection ${ }^{12,40}$. Therefore, it could be inferred that the SARS-CoV-2-S1-RBD and FCS might be the cause for the severity of the infection and in addition, some other factors also contribute to the increased transmissibility of infection in humans which is the hallmark for the SARS-CoV-2 in comparison to SARS-CoV-1 and MERS-CoV.

In comparison to other coronaviruses, SARS-CoV-2 has a large flat, non-sunken sialic acidbinding domain with extended and divergent loop sections of the SARS-CoV-2 S1-NTD is expected to improve viral transmission and infectivity by facilitating sialic acid-binding ability $^{41-45}$. Furthermore, glycosylated cell surface proteins and lipids are in all types of cells and play a role in immunity and cell-cell communication ${ }^{13,14,46-48}$. Perhaps, SARS-CoV-2 can first attach to any type of cells with the S1-NTD to the cell surface sugar receptor. Further, allow the S1-RBD to search for the specific receptor for the virus entry into the cell thereby dramatically increases the transmissibility of infection. The SARS-CoV-2 S1-NTD specific $\mathrm{mAb}$ treatment showed the role of S1-NTD in the receptor post-attachment virus entry (without affecting the binding of the S1-RBD with the ACE2), and cell-cell fusion thereby completely abolish the clinical onset of the infection ${ }^{12,49}$. In addition, most of the highly transmissible variants of SARS-CoV-2 such as Mink Cluster 5, B.1.1.7, B.1.351, P.1, B.1.617.1, B.1.617.2, B.1.526, B.1.525, B.1.177, B.1.427 and B.1.429 were linked to the mutations/deletions in the S1-NTDs in natural infection ${ }^{12,49-56}$ and also in the experimental condition $^{49}$. Furthermore, the Cambodia bat viruses (RShSTT200 and RShSTT182) which are the recombinant of SARS-CoV-2/RatG13 with S1-NTD of SARS-like bat coronavirus were not detected in the ongoing pandemics, while bat/RatG13 (with Type-1-S1-NTD) established pandemic, which indicates the significance of the novel Type-I-S1-NTD in the SARS-CoV-2 genome for its rapid pandemic spread.

The pseudotyped virus of S protein RatG13 has a poor ability to infect many cell types, including bat and human cells ${ }^{23,40}$. The RaTG13 is the only one bat virus RNA sequence having the Type-I-S1-NTD remains unknown. It could be due to (i) Reverse zoonosis of human-specific Type-I-S1-NTD to bats, as SARS-CoV-2 is known to cause reverse zoonosis $^{57-63}$; or (ii) Contamination of human virus to bat sample during sample collection, transport, storage, and sample processing, and this theory might be disproved by isolation of 
the virus and experimental infection; or (iii) The RaTG13 virus may spontaneously evolve in bats; however, the RaTG13 (with Type-I-S1-NTD) and Pangolin/GX/2018 lineage (with Type-I-like-S1-NTD) S gene pseudotyped viruses fail to infect bat cells ${ }^{40}$; whereas, Pangolin/Guangdong/2019 lineage (with TypeII-like-S1-NTD) efficiently infect the bat cells $^{40}$. Therefore, it can be inferred that the Type-I-S1-NTD is the most potential for enhancing human adaptation.

Importantly, we produced evidence of extensive recombination events in bat viruses, particularly in the S1-NTD and S1-RBD areas, and we also confirmed the existence of selection pressure to evolve the S1-NTD region in this study. In light of this, we hypothesize that SARS-CoV-2-rB-CoVs with Type-II-S1-NTD probably jumped to pangolins and then recombination between Type-II-S1-NTD (SARS-CoV-2-rB-CoV) and Type-I-like-S1-NTD (which shares $73 \%$ identity with Type-I-S1-NTD) may have occurred, resulting in the emergence of SARS-CoV-2 related pangolin virus with Type-I-like-S1-NTD. Furthermore, jumping of this virus into the human, and evolved into SARS-CoV-2 with Type-I-S1-NTD due to the host selection pressure. Our results suggest that the pangolins are the promising mixing vessel for bat and human SARS coronaviruses (Fig. 4), as pigs are the mixing vessel for influenza viruses ${ }^{64-67}$.

In conclusion, SARS-CoV-2 gained the novel S1-NTD; probably originated from bat through pangolin mixing vessels by recombination. The identification of the potent host receptor for the different types of S1-NTDs to determine its role in viral spread, host range, and severity of infection needs to be studied. Further, structure-based studies on the importance of mutations in the Type-I-S1-NTD and monitoring of mutations in this domain are warranted, to predict the future direction of the current pandemic; to design novel antiviral molecules, development of vaccines, and neutralizing antibodies to prevent and control the ongoing and future outbreaks.

\section{Materials and Method}

\section{Genetic diversity analysis}

\section{a. Phylogenetic analysis}

The NCBI and GISAID databases were used to find the SARS-CoV-2 and similar bat coronavirus sequences. For the phylogenetic study, MEGA7 was utilized, with the NeighborJoining method being used to infer the evolutionary history, bootstrap tests (1000 replicates), the Maximum Composite Likelihood method being used to compute the evolutionary distances, and gamma distribution (shape parameter $=5$ ) being employed to model the rate variation among sites. Differences in composition bias among sequences were assessed for evolutionary comparisons, and all ambiguous sites were ignored in the analysis for each sequence pair.

\section{b. Net Between Group Mean Distance (NBGMD) analysis}

The NBGMD was calculated using the MEGA7 through the Kimura 2-parameter model, with a gamma distribution (shape parameter $=5$ ) used as a model to measure rate variation among 
sites, a bootstrap test (1000 replicates) used to estimate the Standard error and all ambiguous sites were removed from the analysis for each sequence pair. Standard error estimates were displayed above the diagonal.

\section{Recombination analysis}

\section{a. SimPlot analysis}

The percent identity between the query and reference sequences was determined using the SimPlot 3.5.1 programme ${ }^{68}$. The nucleotide sequences were first aligned in MEGA7 before being exported to SimPlot 3.5.1 for additional analysis. To measure the identity between the query and reference sequences, we employed the 500 base pair of the window at a 50 base pair step, using the Kimura two-parameter model.

\section{b. Recombination Detection Program (RDP) analysis}

RDP4 ${ }^{69}$ was used to detect potential recombination events in SARS-CoV-2 and its related viruses. MEGA7 was used to align the nucleotides, which were subsequently exported to RDP4 for additional processing. The analysis was conducted with default parameter values for the BOOTSCAN, GENECONV, Chimaera, RDP, MaxChi, SISCAN, and 3seq methods, and a minimum of four or more approaches was assessed for probable recombination using a Bonferroni corrected p-value cut-off (0.05). The BOOTSCAN method-based images are displayed in the figures.

\section{Determining the selection and mutation pressure}

\section{a. Effective Number of Codons (ENc)}

One of the approaches for determining the codon usage bias is the effective number of codons used from 61 codons for the 20 amino acids, which might range from 20 to 61. ENc values less than 35 suggest strong codon bias, while ENc values greater than 50 imply broad random codon usage ${ }^{27,70}$. ENc values were calculated using an online server in this investigation (http://ppuigbo.me/programs/CAIcal/ $)^{71}$.

\section{b. ENc-GC3s plot}

The ENc values are plotted against the third position of GC3s of codon values in this study to establish the key variables affecting the codon usage bias, such as selection or mutation pressure $^{28}$. The expected curve was determined by estimating the expected ENc values for each GC3S as recommended in previous publications ${ }^{27,28}$. The ENc and GC3s for every gene were obtained from an online CAI analysis server (http://ppuigbo.me/programs/CAIcal/ $)^{71}$. When codon bias is just driven by mutation pressure, the genes will lie on or near the expected curve, however, when codon bias is influenced by selection and other variables, the genes will fall well below the expected curve. ${ }^{27,28}$. 
313 The GC12 values of codons are plotted against the GC3 values in a neutrality plot to 314 determine the degree of influence of mutation pressure and natural selection on codon usage 315 patterns. The GC12 and GC3 values for S1-NTD (region 6) nucleotide sequences were retrieved from the online CAI analysis server (http://ppuigbo.me/programs/CAIcal//) ${ }^{71}$.

\section{d. Parity Rule 2 (PR2)-bias plot}

To measure the mutation pressure and natural selection affecting the codon use bias, the AT bias $[\mathrm{A} 3 /(\mathrm{A} 3+\mathrm{T} 3)]$ is plotted against the $\mathrm{GC}$ bias $[\mathrm{G} 3 /(\mathrm{G} 3+\mathrm{C} 3)]^{28}$. Using the ACUA Software $^{72}$, the A3, T3, G3, and C3 values of nucleotide sequences of S1-NTD (region 6) were calculated

\section{Funding}

P.A.D is a DST-INSPIRE faculty is supported by research funding from the Government of India (DST/INSPIRE/04/2016/001067). P.A.D is supported by research funding from the Science and Engineering Research Board, Department of Science and Technology, Government of India (CRG/2018/002192).

\section{Acknowledgments}

We thank Director, IISc, Bangalore, India, The Chair, Department of Microbiology and Cell Biology, Indian Institute of Science, Bengaluru, India, and Prof. Nagalingam R. Sundaresan, Department of Microbiology and Cell Biology, Indian Institute of Science, Bengaluru, India for the providing working place at IISc, Bangalore.

\section{Author contributions}

PAD performed most of the bioinformatics experiments. KN assisted PAD for most of the bioinformatics works. PAD and KN wrote the first draft of the manuscript. PAD conceived the study, designed experiments, and wrote the final version of the manuscript.

\section{Conflict of interest: None.}

\section{References}

1 Wu, F. et al. A new coronavirus associated with human respiratory disease in China. Nature 579, 265-269, doi:10.1038/s41586-020-2008-3 (2020).

2 Zhou, P. et al. A pneumonia outbreak associated with a new coronavirus of probable bat origin. Nature 579, 270-273, doi:10.1038/s41586-020-2012-7 (2020).

3 Zhou, H. et al. Identification of novel bat coronaviruses sheds light on the evolutionary origins of SARS-CoV-2 and related viruses. Cell, doi:10.1016/j.cell.2021.06.008 (2021).

4 Zhou, H. et al. A Novel Bat Coronavirus Closely Related to SARS-CoV-2 Contains Natural Insertions at the S1/S2 Cleavage Site of the Spike Protein. Current biology : CB 30, 2196-2203 e2193, doi:10.1016/j.cub.2020.05.023 (2020).

5 Wacharapluesadee, S. et al. Evidence for SARS-CoV-2 related coronaviruses circulating in bats and pangolins in Southeast Asia. Nature communications 12, 972, doi:10.1038/s41467021-21240-1 (2021). 
6 Lam, T. T. et al. Identifying SARS-CoV-2-related coronaviruses in Malayan pangolins. Nature 583, 282-285, doi:10.1038/s41586-020-2169-0 (2020).

7 Xiao, K. et al. Isolation of SARS-CoV-2-related coronavirus from Malayan pangolins. Nature 583, 286-289, doi:10.1038/s41586-020-2313-x (2020).

8 York, A. Searching for relatives of SARS-CoV-2 in bats. Nature reviews. Microbiology, doi:10.1038/s41579-021-00595-8 (2021).

9 Walls, A. C. et al. Structure, Function, and Antigenicity of the SARS-CoV-2 Spike Glycoprotein. Cell 181, 281-292 e286, doi:10.1016/j.cell.2020.02.058 (2020).

$10 \mathrm{Chi}, \mathrm{X}$. et al. A neutralizing human antibody binds to the $\mathrm{N}$-terminal domain of the Spike protein of SARS-CoV-2. Science 369, 650-655, doi:10.1126/science.abc6952 (2020).

11 Greaney, A. J. et al. Complete Mapping of Mutations to the SARS-CoV-2 Spike ReceptorBinding Domain that Escape Antibody Recognition. Cell host \& microbe 29, 44-57 e49, doi:10.1016/j.chom.2020.11.007 (2021).

12 Suryadevara, N. et al. Neutralizing and protective human monoclonal antibodies recognizing the N-terminal domain of the SARS-CoV-2 spike protein. Cell 184, 2316-2331 e2315, doi:10.1016/j.cell.2021.03.029 (2021).

13 Li, F. Structure, Function, and Evolution of Coronavirus Spike Proteins. Annual review of virology 3, 237-261, doi:10.1146/annurev-virology-110615-042301 (2016).

14 Li, F. Receptor recognition mechanisms of coronaviruses: a decade of structural studies. Journal of virology 89, 1954-1964, doi:10.1128/JVI.02615-14 (2015).

15 Hulswit, R. J., de Haan, C. A. \& Bosch, B. J. Coronavirus Spike Protein and Tropism Changes. Advances in virus research 96, 29-57, doi:10.1016/bs.aivir.2016.08.004 (2016).

16 Wang, Q. et al. Structural and Functional Basis of SARS-CoV-2 Entry by Using Human ACE2. Cell 181, 894-904 e899, doi:10.1016/j.cell.2020.03.045 (2020).

17 Huang, Y., Yang, C., Xu, X. F., Xu, W. \& Liu, S. W. Structural and functional properties of SARSCoV-2 spike protein: potential antivirus drug development for COVID-19. Acta pharmacologica Sinica 41, 1141-1149, doi:10.1038/s41401-020-0485-4 (2020).

$18 \mathrm{Li}, \mathrm{F}$. et al. Conformational states of the severe acute respiratory syndrome coronavirus spike protein ectodomain. Journal of virology 80, 6794-6800, doi:10.1128/JVI.02744-05 (2006).

19 Beniac, D. R., Andonov, A., Grudeski, E. \& Booth, T. F. Architecture of the SARS coronavirus prefusion spike. Nature structural \& molecular biology 13, 751-752, doi:10.1038/nsmb1123 (2006).

20 Watanabe, Y., Allen, J. D., Wrapp, D., McLellan, J. S. \& Crispin, M. Site-specific glycan analysis of the SARS-CoV-2 spike. Science 369, 330-333, doi:10.1126/science.abb9983 (2020).

21 Piccoli, L. et al. Mapping Neutralizing and Immunodominant Sites on the SARS-CoV-2 Spike Receptor-Binding Domain by Structure-Guided High-Resolution Serology. Cell 183, 10241042 e1021, doi:10.1016/j.cell.2020.09.037 (2020).

22 Rogers, T. F. et al. Isolation of potent SARS-CoV-2 neutralizing antibodies and protection from disease in a small animal model. Science 369, 956-963, doi:10.1126/science.abc7520 (2020).

23 Wrobel, A. G. et al. SARS-CoV-2 and bat RaTG13 spike glycoprotein structures inform on virus evolution and furin-cleavage effects. Nature structural \& molecular biology 27, 763767, doi:10.1038/s41594-020-0468-7 (2020).

$24 \mathrm{Ou}, \mathrm{X}$. et al. Characterization of spike glycoprotein of SARS-CoV-2 on virus entry and its immune cross-reactivity with SARS-CoV. Nature communications 11, 1620, doi:10.1038/s41467-020-15562-9 (2020).

25 Hon, C. C. et al. Evidence of the recombinant origin of a bat severe acute respiratory syndrome (SARS)-like coronavirus and its implications on the direct ancestor of SARS coronavirus. Journal of virology 82, 1819-1826, doi:10.1128/JVI.01926-07 (2008).

26 Lin, X. D. et al. Extensive diversity of coronaviruses in bats from China. Virology 507, 1-10, doi:10.1016/j.virol.2017.03.019 (2017). 
27 Wang, L. et al. Genome-wide analysis of codon usage bias in four sequenced cotton species. PLoS One 13, e0194372, doi:10.1371/journal.pone.0194372 (2018).

28 Tian, H. F. et al. Genetic and codon usage bias analyses of major capsid protein gene in Ranavirus. Infect Genet Evol 84, 104379, doi:10.1016/j.meegid.2020.104379 (2020).

29 Wang, N. et al. Structure of MERS-CoV spike receptor-binding domain complexed with human receptor DPP4. Cell research 23, 986-993, doi:10.1038/cr.2013.92 (2013).

30 Coutard, B. et al. The spike glycoprotein of the new coronavirus 2019-nCoV contains a furinlike cleavage site absent in CoV of the same clade. Antiviral research 176, 104742, doi:10.1016/j.antiviral.2020.104742 (2020).

31 Ramanathan, M., Ferguson, I. D., Miao, W. \& Khavari, P. A. SARS-CoV-2 B.1.1.7 and B.1.351 spike variants bind human ACE2 with increased affinity. The Lancet. Infectious diseases, doi:10.1016/S1473-3099(21)00262-0 (2021).

32 Andersen, K. G., Rambaut, A., Lipkin, W. I., Holmes, E. C. \& Garry, R. F. The proximal origin of SARS-CoV-2. Nature medicine 26, 450-452, doi:10.1038/s41591-020-0820-9 (2020).

33 Zhang, T., Wu, Q. \& Zhang, Z. Probable Pangolin Origin of SARS-CoV-2 Associated with the COVID-19 Outbreak. Current biology : CB 30, 1346-1351 e1342, doi:10.1016/j.cub.2020.03.022 (2020).

34 Zhang, Y. Z. \& Holmes, E. C. A Genomic Perspective on the Origin and Emergence of SARSCoV-2. Cell 181, 223-227, doi:10.1016/j.cell.2020.03.035 (2020).

35 Segreto, R. \& Deigin, Y. The genetic structure of SARS-CoV-2 does not rule out a laboratory origin: SARS-COV-2 chimeric structure and furin cleavage site might be the result of genetic manipulation. BioEssays : news and reviews in molecular, cellular and developmental biology 43, e2000240, doi:10.1002/bies.202000240 (2021).

$36 \mathrm{Wu}, \mathrm{Y}$. \& Zhao, S. Furin cleavage sites naturally occur in coronaviruses. Stem cell research $\mathbf{5 0}$, 102115, doi:10.1016/j.scr.2020.102115 (2020).

37 Xia, S. et al. The role of furin cleavage site in SARS-CoV-2 spike protein-mediated membrane fusion in the presence or absence of trypsin. Signal transduction and targeted therapy 5, 92, doi:10.1038/s41392-020-0184-0 (2020).

38 Lan, J. et al. Structure of the SARS-CoV-2 spike receptor-binding domain bound to the ACE2 receptor. Nature 581, 215-220, doi:10.1038/s41586-020-2180-5 (2020).

39 Shang, J. et al. Structural basis of receptor recognition by SARS-CoV-2. Nature 581, 221-224, doi:10.1038/s41586-020-2179-y (2020).

$40 \mathrm{Nie}, \mathrm{J}$. et al. Functional comparison of SARS-CoV-2 with closely related pangolin and bat coronaviruses. Cell discovery 7, 21, doi:10.1038/s41421-021-00256-3 (2021).

41 Lundstrom, K. et al. Viewpoint: Origin of SARS-CoV-2. Viruses 12, doi:10.3390/v12111203 (2020).

42 Vandelli, A. et al. Structural analysis of SARS-CoV-2 genome and predictions of the human interactome. Nucleic acids research 48, 11270-11283, doi:10.1093/nar/gkaa864 (2020).

43 Baker, A. N. et al. The SARS-COV-2 Spike Protein Binds Sialic Acids and Enables Rapid Detection in a Lateral Flow Point of Care Diagnostic Device. ACS central science 6, 20462052, doi:10.1021/acscentsci.0c00855 (2020).

44 Seyran, M. et al. The structural basis of accelerated host cell entry by SARS-CoV-2dagger. The FEBS journal, doi:10.1111/febs.15651 (2020).

45 Sun, X. L. The Role of Cell Surface Sialic Acids for SARS-CoV-2 Infection. Glycobiology, doi:10.1093/glycob/cwab032 (2021).

46 Dove, A. The bittersweet promise of glycobiology. Nature biotechnology 19, 913-917, doi:10.1038/nbt1001-913 (2001).

47 Ghazarian, H., Idoni, B. \& Oppenheimer, S. B. A glycobiology review: carbohydrates, lectins and implications in cancer therapeutics. Acta histochemica 113, 236-247, doi:10.1016/j.acthis.2010.02.004 (2011). 
490

491

492

493

494

495

496

497

498

499 500

48 Schwegmann-Wessels, C. \& Herrler, G. Sialic acids as receptor determinants for coronaviruses. Glycoconjugate journal 23, 51-58, doi:10.1007/s10719-006-5437-9 (2006).

49 McCallum, M. et al. N-terminal domain antigenic mapping reveals a site of vulnerability for SARS-CoV-2. Cell 184, 2332-2347 e2316, doi:10.1016/j.cell.2021.03.028 (2021).

50 McCarthy, K. R. et al. Recurrent deletions in the SARS-CoV-2 spike glycoprotein drive antibody escape. Science 371, 1139-1142, doi:10.1126/science.abf6950 (2021).

51 McCallum, M. et al. SARS-CoV-2 immune evasion by variant B.1.427/B.1.429. bioRxiv, doi:10.1101/2021.03.31.437925 (2021).

52 Annavajhala, M. K. et al. A Novel SARS-CoV-2 Variant of Concern, B.1.526, Identified in New York. medRxiv : the preprint server for health sciences, doi:10.1101/2021.02.23.21252259 (2021).

53 Hodcroft, E. B. et al. Spread of a SARS-CoV-2 variant through Europe in the summer of 2020. Nature, doi:10.1038/s41586-021-03677-y (2021).

54 Kemp, S. A. et al. SARS-CoV-2 evolution during treatment of chronic infection. Nature 592, 277-282, doi:10.1038/s41586-021-03291-y (2021).

55 Meng, B. et al. Recurrent emergence of SARS-CoV-2 spike deletion H69/V70 and its role in the Alpha variant B.1.1.7. Cell reports 35, 109292, doi:10.1016/j.celrep.2021.109292 (2021).

56 Avanzato, V. A. et al. Case Study: Prolonged Infectious SARS-CoV-2 Shedding from an Asymptomatic Immunocompromised Individual with Cancer. Cell 183, 1901-1912 e1909, doi:10.1016/j.cell.2020.10.049 (2020).

57 Sit, T. H. C. et al. Infection of dogs with SARS-CoV-2. Nature 586, 776-778, doi:10.1038/s41586-020-2334-5 (2020).

58 Oude Munnink, B. B. et al. Transmission of SARS-CoV-2 on mink farms between humans and mink and back to humans. Science 371, 172-177, doi:10.1126/science.abe5901 (2021).

59 Shi, J. et al. Susceptibility of ferrets, cats, dogs, and other domesticated animals to SARScoronavirus 2. Science 368, 1016-1020, doi:10.1126/science.abb7015 (2020).

60 Richard, M. et al. SARS-CoV-2 is transmitted via contact and via the air between ferrets. Nature communications 11, 3496, doi:10.1038/s41467-020-17367-2 (2020).

61 Sia, S. F. et al. Pathogenesis and transmission of SARS-CoV-2 in golden hamsters. Nature 583, 834-838, doi:10.1038/s41586-020-2342-5 (2020).

62 Oreshkova, N. et al. SARS-CoV-2 infection in farmed minks, the Netherlands, April and May 2020. Euro surveillance : bulletin Europeen sur les maladies transmissibles = European communicable disease bulletin 25, doi:10.2807/1560-7917.ES.2020.25.23.2001005 (2020).

63 McAloose, D. et al. From People to Panthera: Natural SARS-CoV-2 Infection in Tigers and Lions at the Bronx Zoo. mBio 11, doi:10.1128/mBio.02220-20 (2020).

64 Taubenberger, J. K. \& Kash, J. C. Influenza virus evolution, host adaptation, and pandemic formation. Cell host \& microbe 7, 440-451, doi:10.1016/j.chom.2010.05.009 (2010).

65 Garten, R. J. et al. Antigenic and genetic characteristics of swine-origin 2009 A(H1N1) influenza viruses circulating in humans. Science 325, 197-201, doi:10.1126/science.1176225 (2009).

66 Ma, W., Kahn, R. E. \& Richt, J. A. The pig as a mixing vessel for influenza viruses: Human and veterinary implications. Journal of molecular and genetic medicine : an international journal of biomedical research 3, 158-166 (2008).

67 Hass, J., Matuszewski, S., Cieslik, D. \& Haase, M. The role of swine as "mixing vessel" for interspecies transmission of the influenza A subtype H1N1: a simultaneous Bayesian inference of phylogeny and ancestral hosts. Infection, genetics and evolution : journal of molecular epidemiology and evolutionary genetics in infectious diseases 11, 437-441, doi:10.1016/j.meegid.2010.12.001 (2011).

68 Paraskevis, D. et al. Full-genome evolutionary analysis of the novel corona virus (2019-nCoV) rejects the hypothesis of emergence as a result of a recent recombination event. Infection, 
genetics and evolution : journal of molecular epidemiology and evolutionary genetics in infectious diseases 79, 104212, doi:10.1016/j.meegid.2020.104212 (2020).

69 Martin, D. P., Murrell, B., Golden, M., Khoosal, A. \& Muhire, B. RDP4: Detection and analysis of recombination patterns in virus genomes. Virus evolution 1, vev003, doi:10.1093/ve/vev003 (2015).

70 Zhao, Y. et al. Analysis of codon usage bias of envelope glycoprotein genes in nuclear polyhedrosis virus (NPV) and its relation to evolution. BMC Genomics 17, 677, doi:10.1186/s12864-016-3021-7 (2016).

71 Puigbo, P., Bravo, I. G. \& Garcia-Vallve, S. CAlcal: a combined set of tools to assess codon usage adaptation. Biol Direct 3, 38, doi:10.1186/1745-6150-3-38 (2008).

72 Vetrivel, U., Arunkumar, V. \& Dorairaj, S. ACUA: a software tool for automated codon usage analysis. Bioinformation 2, 62-63, doi:10.6026/97320630002062 (2007).

\section{4}

\section{5}

6

17

.

\section{9}

0

1

2



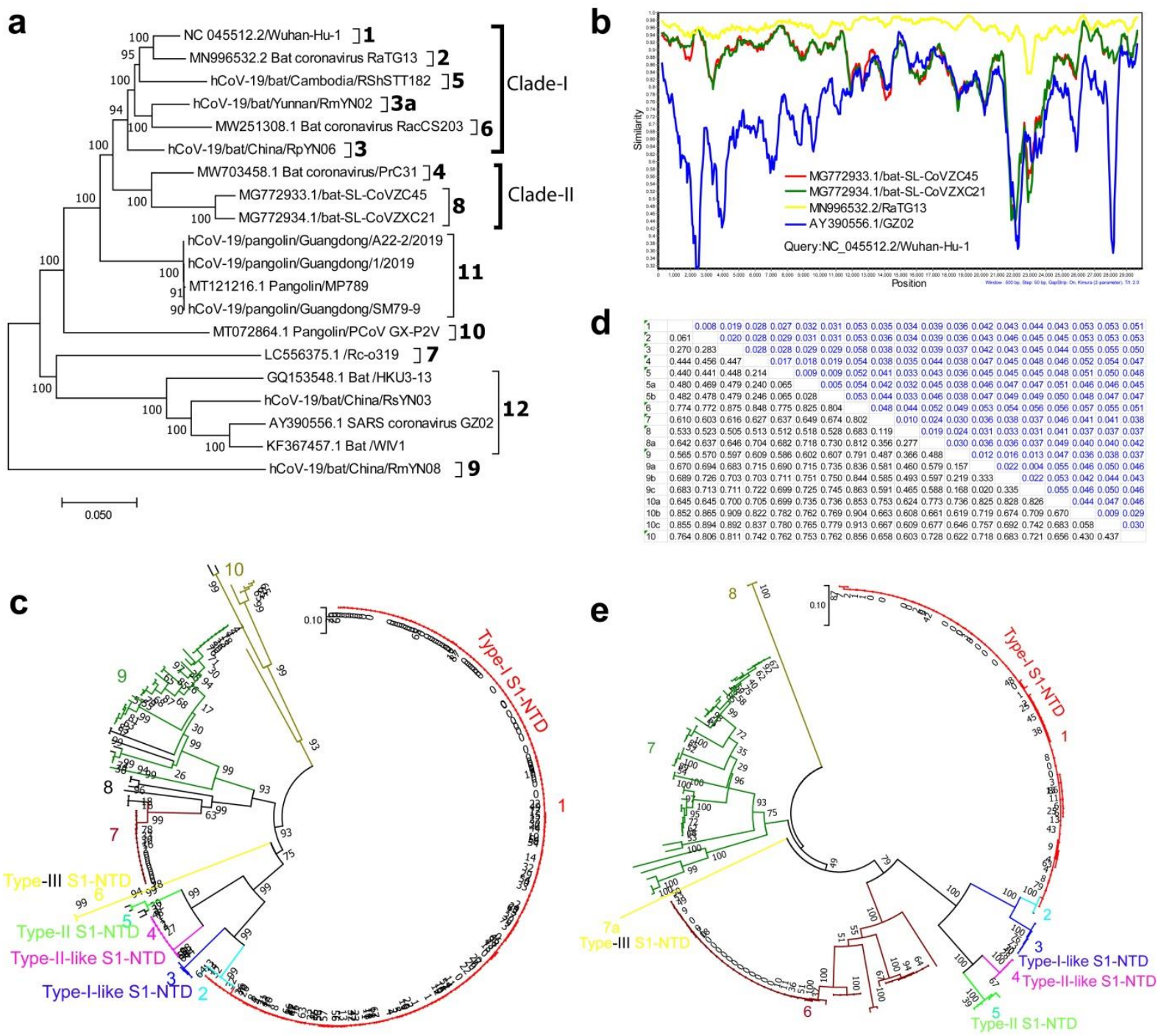

Fig. 1: The S1-NTD based genetic diversity in SARS-CoV-2. a, The Phylogenetic tree using the complete genome's nucleotide sequences of the SARS-CoV-2 and related viruses. b, Similarity plot based on the complete genomes nucleotide sequences of the SARS-CoV-2, RatG13, bat-SL-CoVZC45, bat-SL-CoVZXC21, and SARS-CoV GZ02; SARS-CoV-2 used as a reference sequence. $\mathbf{c} \& \mathbf{d}$, The region 6 (21578-22560nt) specific phylogenetic tree and net between-group mean distance respectively for SARS-CoV-2 related viruses. e, The region 6 specific amino acid-based phylogenetic tree for SARS-CoV-2 related viruses. 


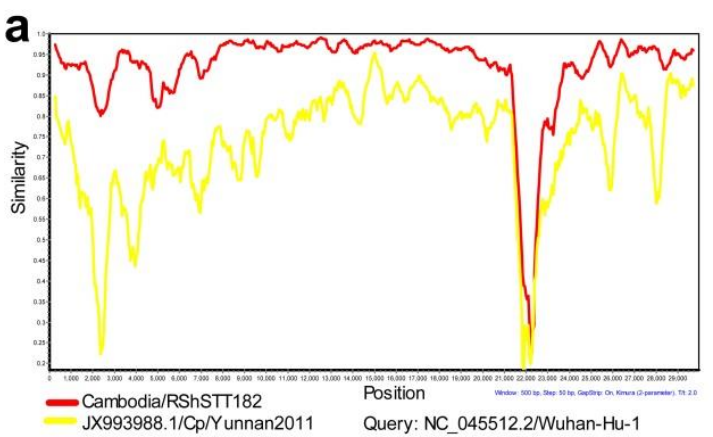

C

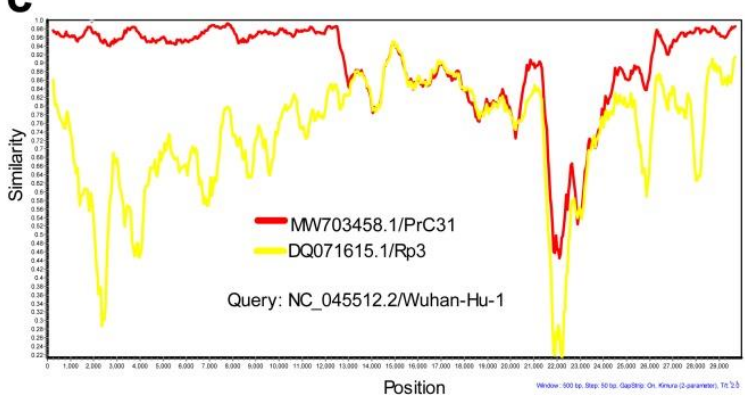

b

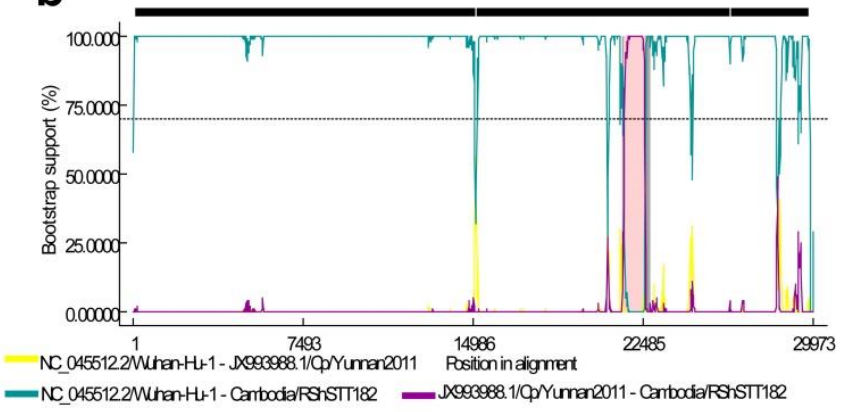

d

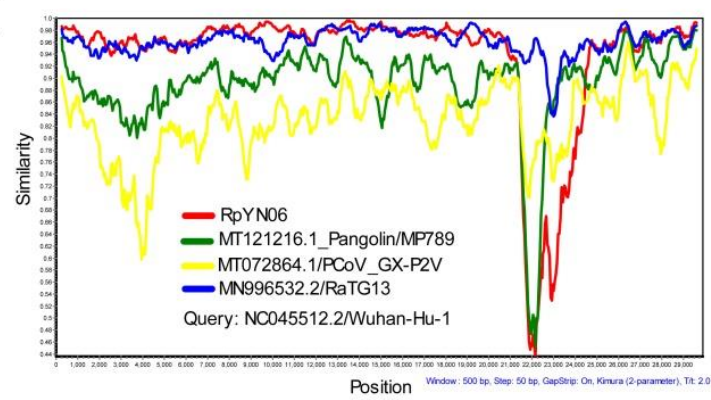

Figure 2

Fig. 2: Recombinant events in the SARS-CoV-2-rB-CoVs. a, Similarity plot based on the complete genomes nucleotide sequences of the SARS-CoV-2, Cambodia bat virus RShSTT182 and Bat coronavirus Cp/Yunnan2011; SARS-CoV-2 used as a reference sequence. b, The recombination analysis based on BOOTSCAN using complete genomes nucleotide sequences of the SARS-CoV-2, Cambodia bat virus RShSTT182 and Bat coronavirus Cp/Yunnan2011; SARS-CoV-2 used as a reference sequence. c, Similarity plot based on the complete genomes nucleotide sequences of the SARS-CoV-2, bat coronavirus PrC31, and Bat SARS coronavirus Rp3; SARS-CoV-2 used as a reference sequence. d, Similarity plot based on the complete genomes nucleotide sequences of the SARS-CoV-2, RpYN06, RatG13 Pangolin/GX/2018, and Pangolin/Guangdong/2019; SARS-CoV-2 used as a reference sequence. 
a
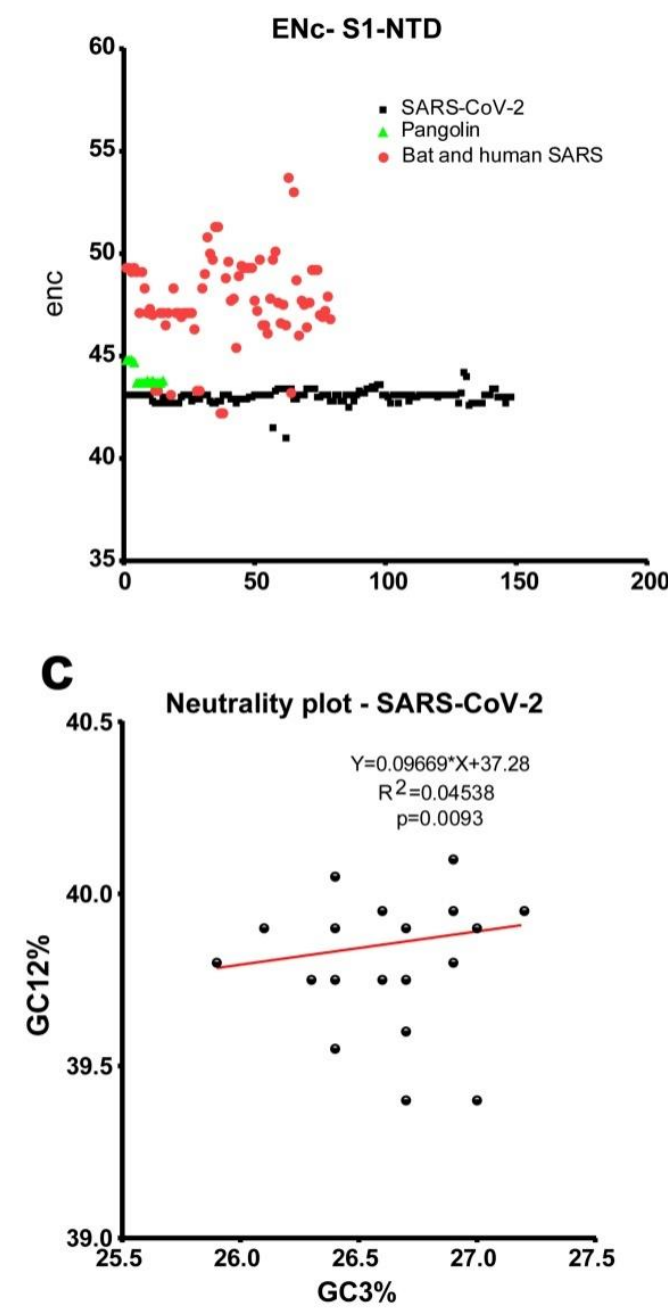

b

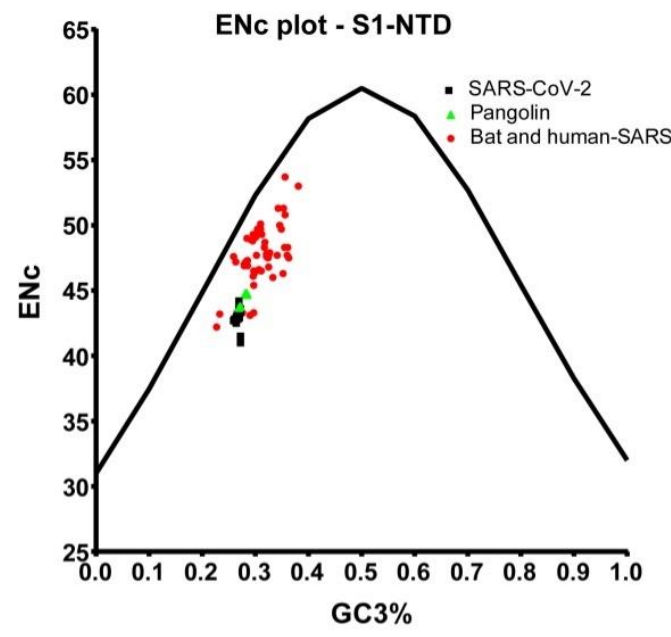

d

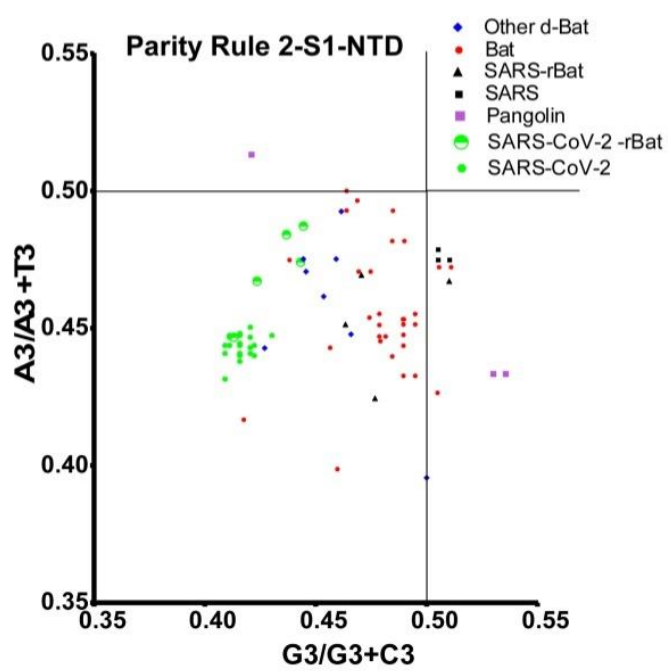

Figure 3

567 Fig. 3: Selection pressure in the S1-NTDs. a, ENc values; b, ENc plotted against GC3s; c, 568 Neutrality plot analysis of the GC12 and that of the GC3; and d, Parity Rule 2(PR2)-bias plot analysis of the SARS-CoV-2, SARS-Cov-2-rB-CoVs, Pangolin-CoVs, SARS-CoVs, and bat SARS viruses. 


\section{a}

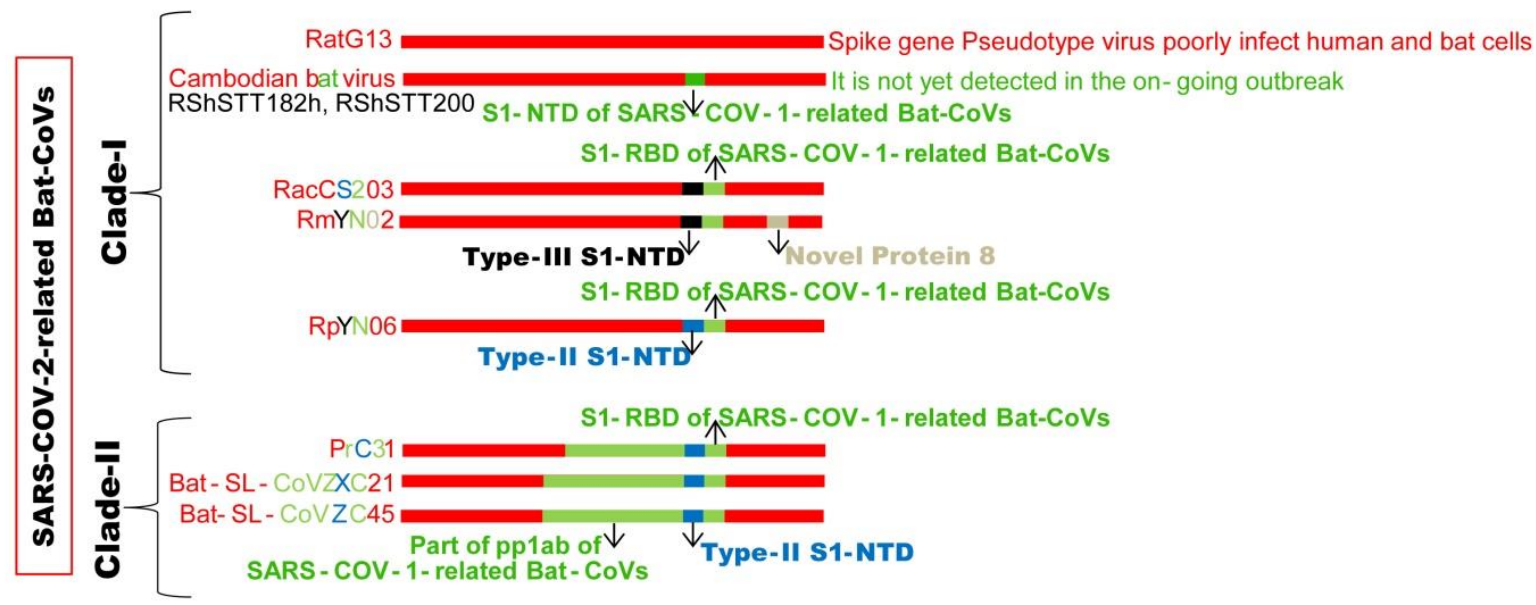

b
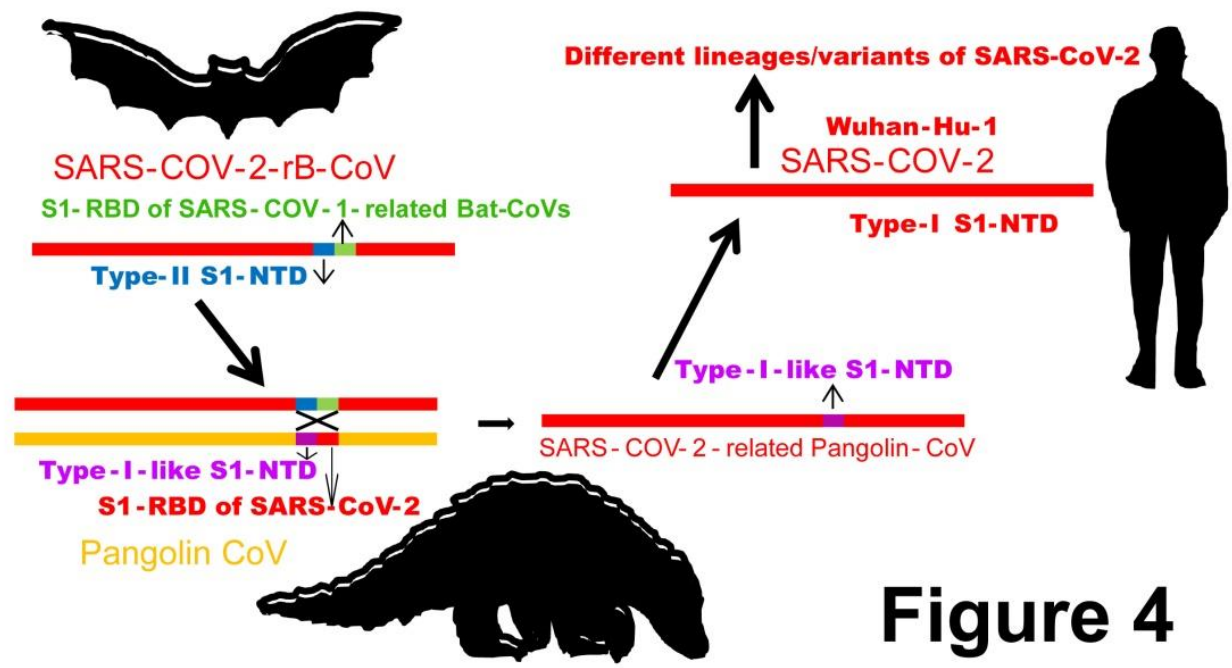

579 Fig.4: A proposed schematic representation of the origin of the Type-I-S1-NTD in

580 SARS-CoV-2. a, The schematic figure explains the classification and the recombination of the SARS-Cov-2-rB-CoVs. b, The schematic figure explains the origin of the SARS-CoV-2 specific Type-I-S1-NTD from the bat-specific Type-II-S1-NTD through the pangolin mixing vessel. The red color represents the SARS-CoV-2 related genomic regions; the green color represents the SARS-CoVs (SARS-Cov-2-rB-CoVs) related genomic regions; The purple color represents the Type-I-like-S1-NTDs; the blue color represents the Type-II-S1-NTDs, and the yellow color represents the pangolin virus. 
0.0010 .0010 .0010 .0010 .0020 .0010 .0040 .0020 .0040 .0020 .0020 .003

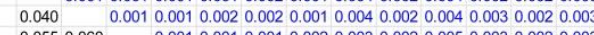
$\begin{array}{llllll}0.055 & 0.060 \quad 0.0010 .0010 .0010 .002 & 0.0030 .002 & 0.005 & 0.003 & 0.0020 .003\end{array}$ 3a $0.0640 .0720 .056 \quad 0.0020 .0010 .0010 .0040 .0020 .0030 .0020 .0020 .003$

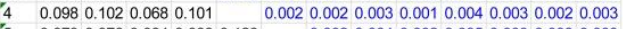

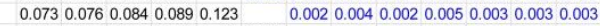
$0.0870 .0890 .0850 .0650 .1210 .092 \quad 0.0040 .0020 .0040 .0030 .0020 .003$

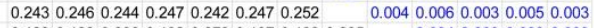
$\begin{array}{llllllllllll}0.122 & 0.122 & 0.096 & 0.132 & 0.072 & 0.137 & 0.132 & 0.235 & 0.004 & 0.003 & 0.002 & 0.003\end{array}$

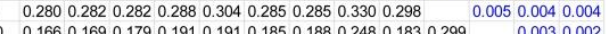

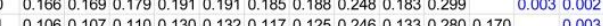
0.1980 .2000 .1950 .2020 .1630 .197020002130 .1570 .2780 .17060 .204

C

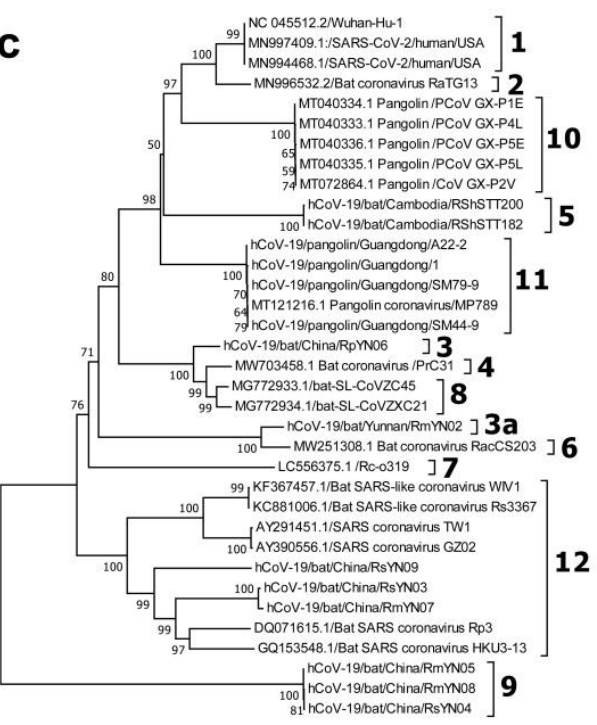

$\stackrel{50.50}{10}$

f

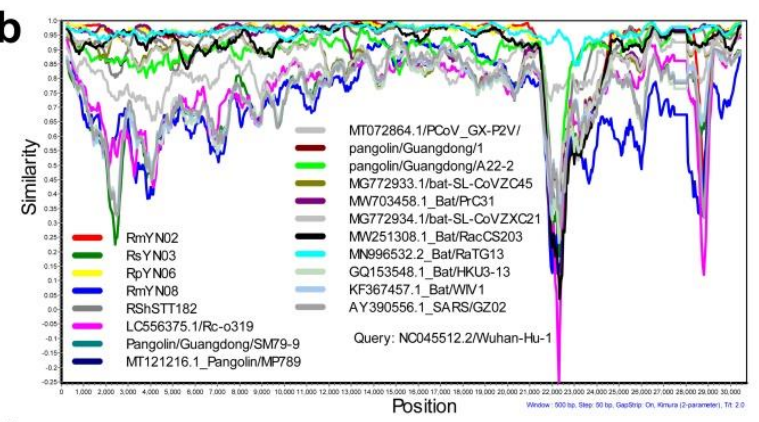

d

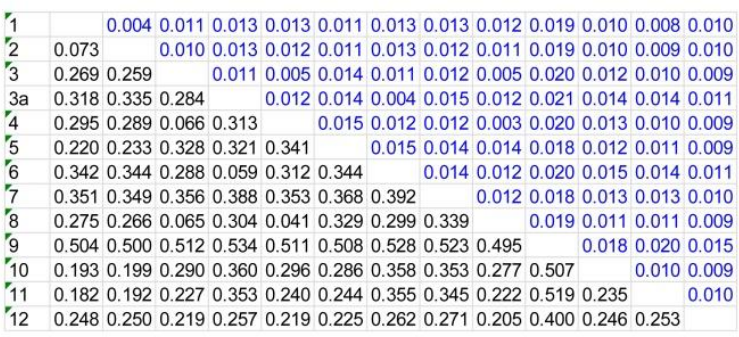

e

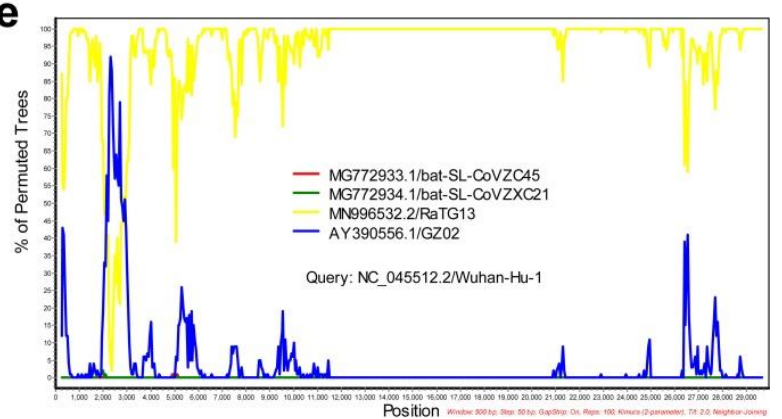
$79 \%$
$82 \%$
$80 \%$
$90 \%$ Identity

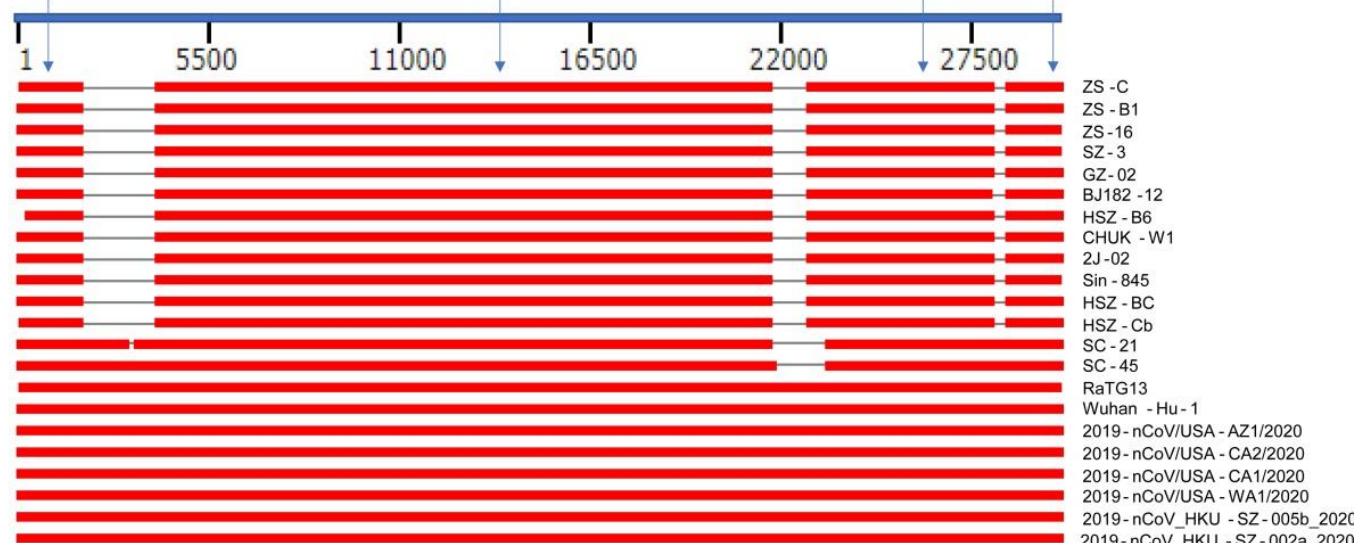

Extended Data Fig. 1

Extended Data Fig. 1: The genetic diversity in the SARS-CoV-2 and related viruses. a,

The net between-group mean distance of the SARS-CoV-2 and related viruses using complete genomes nucleotide sequences. b, Similarity plot based on the complete genomes nucleotide sequences of the SARS-CoV-2 and related viruses. c, The Phylogenetic tree using the complete Spike gene nucleotide sequences of the SARS-CoV-2 and related viruses. d,

7 The net between-group mean distance of the SARS-CoV-2 and related viruses using 8 complete Spike gene nucleotide sequences. e, The BootScan results are based on the 9 complete genomes nucleotide sequences of the SARS-CoV-2, bat-SL-CoVZXC21, bat-SL10 CoVZC45, RaTG13, and SARS-CoV GZ02. The NC_045512.2/Wuhan-Hu-1 virus has been used as a reference sequences query. f, NCBI public database BLAST analyses of the complete genome of Wuhan seafood market pneumonia virus isolate Wuhan-Hu-1 (NC_045512.2), showed the different region-specific nucleotide diversity with the other SARS-CoVs and bat SARSr-CoVs. 

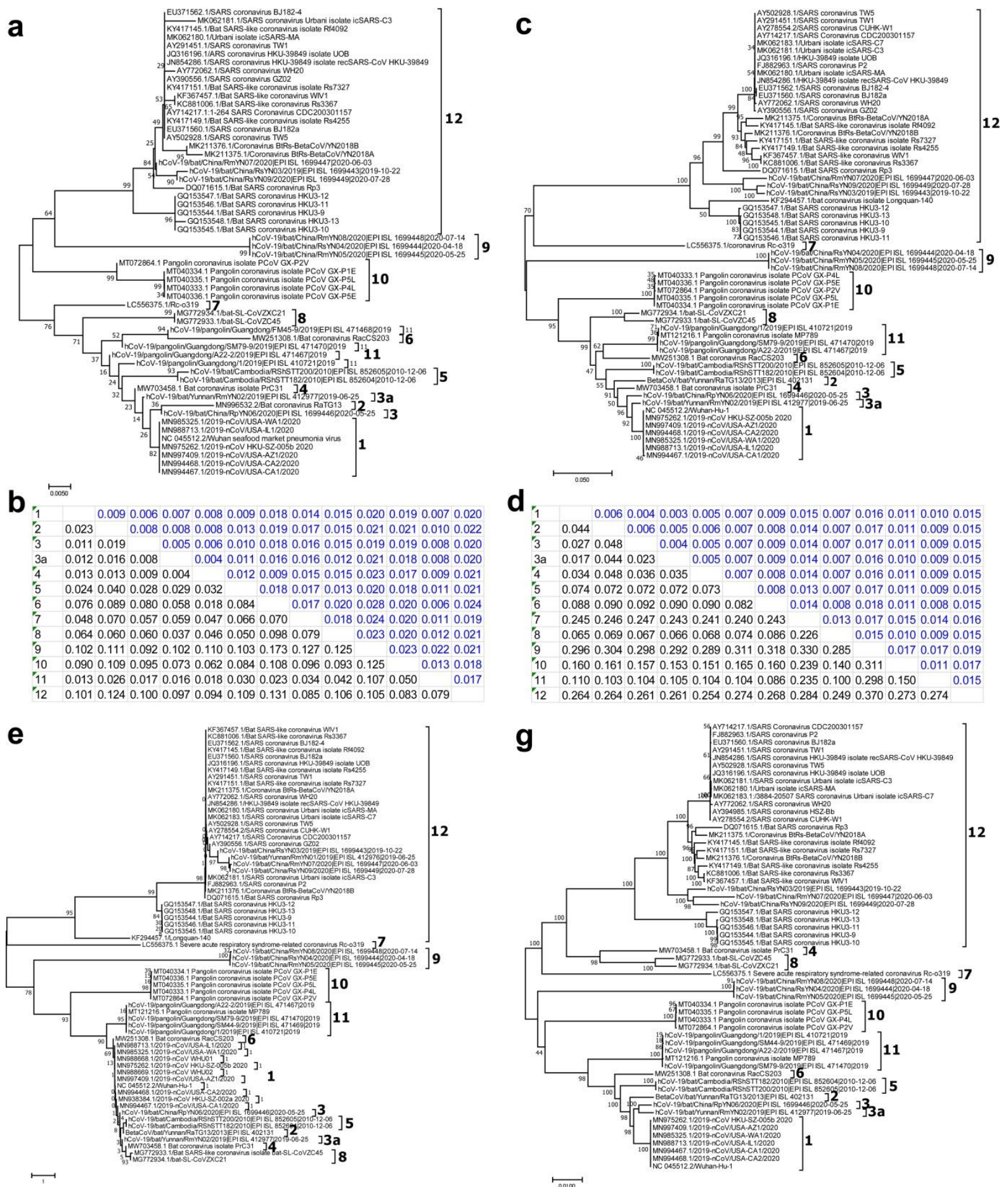
f $\quad 0.0060 .0050 .0050 .0050 .0080 .0060 .0220 .0080 .0220 .0140 .0110 .020$ $\begin{array}{lllllllllllllllllllllll}2 & 0.063 & 0.005 & 0.006 & 0.006 & 0.008 & 0.006 & 0.021 & 0.007 & 0.024 & 0.014 & 0.011 & 0.020\end{array}$

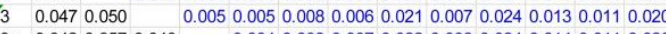 3a $0.0420 .0570 .040 \quad 0.0040 .0080 .0070 .0220 .0080 .0240 .0140 .0110 .020$

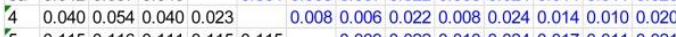 $\begin{array}{llllllllllll}5 & 0.115 & 0.116 & 0.111 & 0.115 & 0.115 & 0.009 & 0.022 & 0.010 & 0.024 & 0.017 & 0.0110 .021\end{array}$

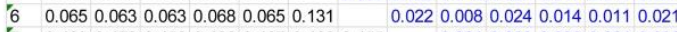

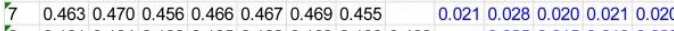 $\begin{array}{lllllllllllll}8 & 0.101 & 0.104 & 0.100 & 0.105 & 0.102 & 0.163 & 0.106 & 0.460 & 0.025 & 0.015 & 0.010 & 0.022\end{array}$ $\begin{array}{llllllllllllllll}9 & 0.505 & 0.508 & 0.517 & 0.525 & 0.518 & 0.529 & 0.509 & 0.553 & 0.507 & 0.025 & 0.024 & 0.027\end{array}$

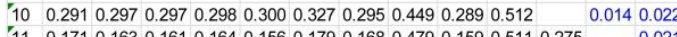 $\begin{array}{lllllllllllllllllllllll}11 & 0.171 & 0.163 & 0.161 & 0.164 & 0.156 & 0.179 & 0.168 & 0.479 & 0.159 & 0.511 & 0.275 & 0.021\end{array}$

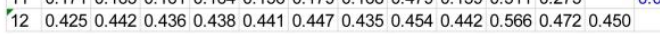
h 1 . 0.0010 .0010 .0020 .0020 .0020 .0030 .0050 .0030 .0040 .0040 .0030 .004

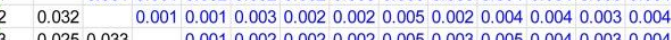 $\begin{array}{llllllllllllll}3 & 0.025 & 0.033 & 0.001 & 0.002 & 0.002 & 0.002 & 0.005 & 0.003 & 0.005 & 0.004 & 0.003 & 0.004\end{array}$ $\begin{array}{lllllllllllll}3 a & 0.027 & 0.035 & 0.020 & 0.002 & 0.002 & 0.002 & 0.005 & 0.003 & 0.004 & 0.004 & 0.003 & 0.004\end{array}$ $\begin{array}{lllllllllllll}4 & 0.088 & 0.093 & 0.082 & 0.081 & 0.003 & 0.003 & 0.004 & 0.002 & 0.005 & 0.004 & 0.004 & 0.003\end{array}$

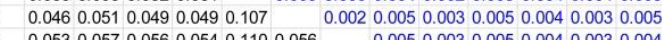 $\begin{array}{llllllllllllllllllll}6 & 0.053 & 0.057 & 0.056 & 0.054 & 0.110 & 0.056 & 0.005 & 0.003 & 0.005 & 0.004 & 0.003 & 0.004\end{array}$

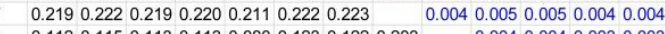

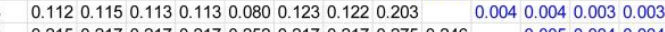 $\begin{array}{llllllllllllllll}9 & 0.215 & 0.217 & 0.217 & 0.217 & 0.252 & 0.217 & 0.217 & 0.275 & 0.246 & 0.005 & 0.004 & 0.004\end{array}$ $\begin{array}{lllllllllllllll}10 & 0.167 & 0.169 & 0.168 & 0.169 & 0.185 & 0.174 & 0.170 & 0.222 & 0.178 & 0.239 & 0.004 & 0.004\end{array}$
$\begin{array}{llllllllllllllll}11 & 0.099 & 0.100 & 0.099 & 0.101 & 0.133 & 0.102 & 0.099 & 0.221 & 0.133 & 0.212 & 0.166 & 0.004\end{array}$

\section{Extended Data Fig. 2}

17 Extended Data Fig. 2: The genetic relationship of SARS-CoV-2 and related viruses in the region $1,2,3$, and 4 . a $\& \mathbf{b}$, The region 1 (5'URT) specific phylogenetic tree and net between-group mean distance respectively for SARS-CoV-2 related viruses. c \& d, The region 2 (266 to $1917 \mathrm{nt}$ ) specific phylogenetic tree and net between-group mean distance respectively for SARS-CoV-2 related viruses. e \& f, The region 3 (1924 -3956nt) specific phylogenetic tree and net between-group mean distance respectively for SARS-CoV-2 related 

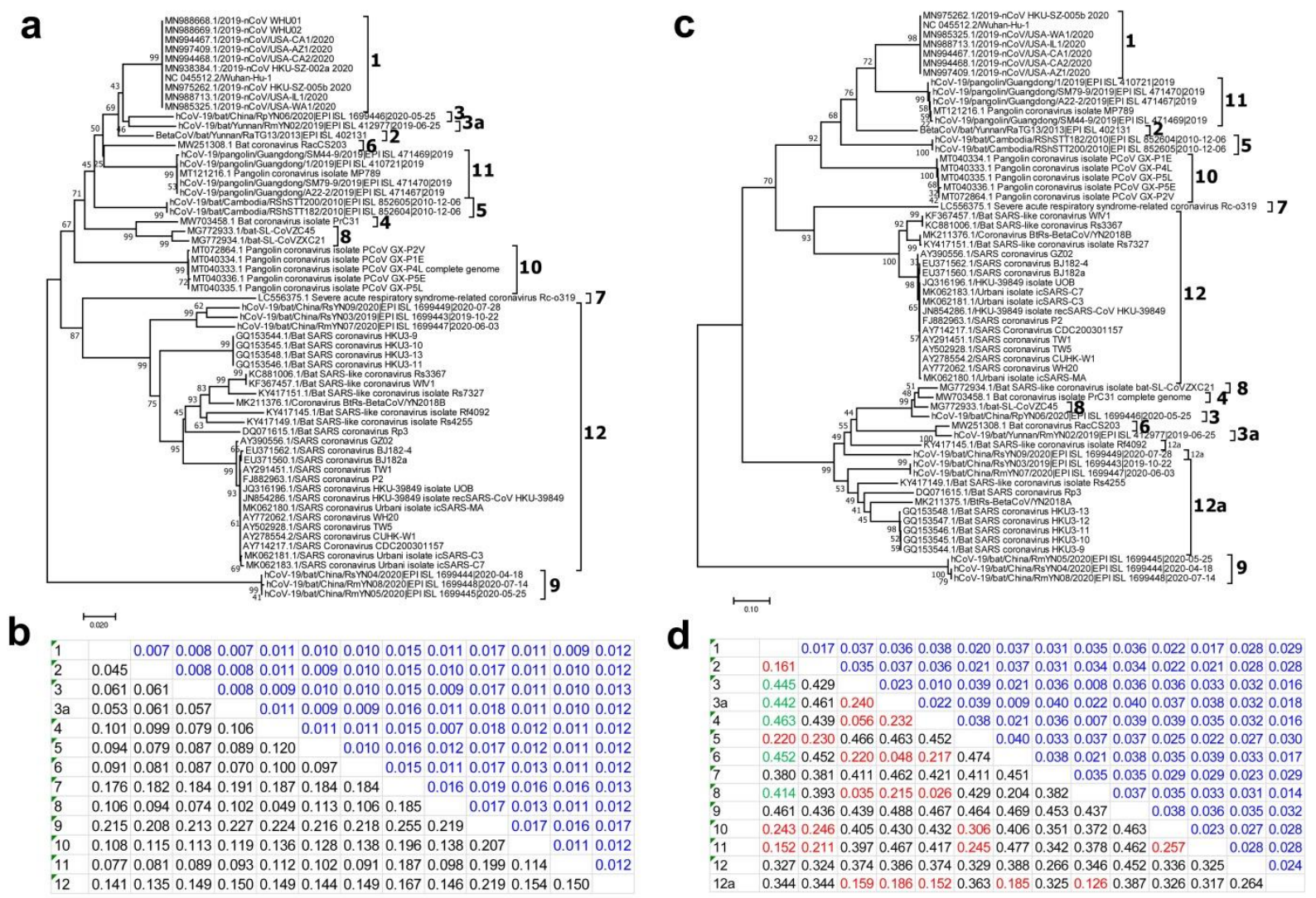

e

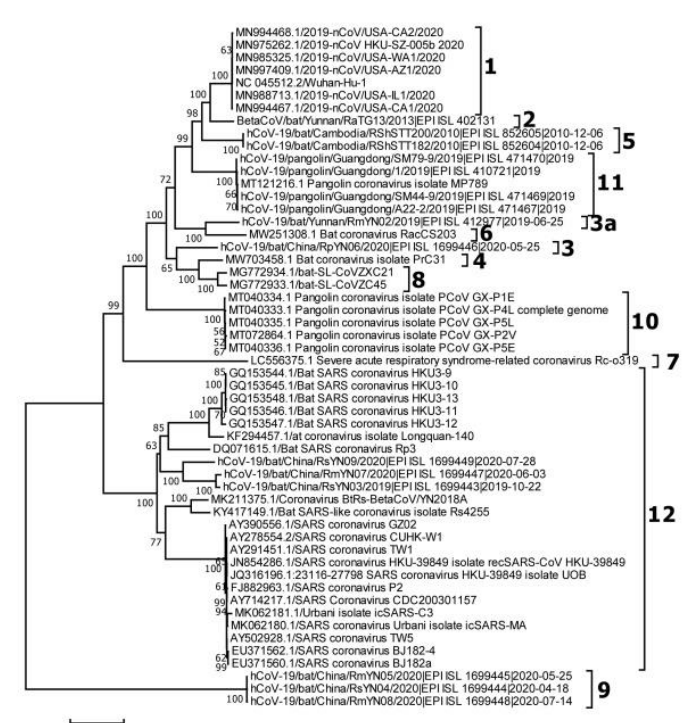

f

$1 \quad 0.0030 .0050 .0050 .0060 .0030 .0050 .0080 .0060 .0120 .0050 .0040 .007$ $\begin{array}{lllllllllllllllllllll}2 & 0.042 & 0.005 & 0.005 & 0.006 & 0.003 & 0.006 & 0.008 & 0.006 & 0.012 & 0.005 & 0.004 & 0.007\end{array}$

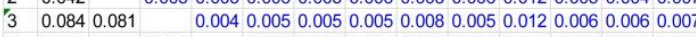
3a $0.0890 .0950 .088 \quad 0.0060 .0050 .0040 .0080 .0070 .0130 .0070 .0060 .007$ $\begin{array}{lllllllllllllllllllllll}4 & 0.116 & 0.116 & 0.081 & 0.137 & 0.006 & 0.006 & 0.009 & 0.003 & 0.011 & 0.006 & 0.007 & 0.007\end{array}$ $\begin{array}{llllllllllllll}5 & 0.065 & 0.071 & 0.103 & 0.110 & 0.130 & 0.006 & 0.006 & 0.007 & 0.005 & 0.012 & 0.006 & 0.005 & 0.007\end{array}$ $\begin{array}{llllllllllll}0.113 & 0.113 & 0.115 & 0.069 & 0.143 & 0.123 & 0.009 & 0.007 & 0.014 & 0.007 & 0.006 & 0.008\end{array}$ $\begin{array}{llllllllllll}0.200 & 0.199 & 0.198 & 0.213 & 0.208 & 0.201 & 0.220 & 0.008 & 0.011 & 0.009 & 0.007 & 0.007\end{array}$ $80.1210 .1160 .0770 .1390 .0400 .1290 .1390 .205 \quad 0.0110 .0060 .0070 .007$

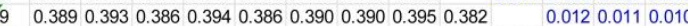

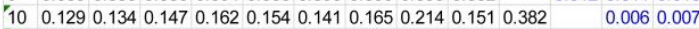
$\begin{array}{llllllllllllllllllllll}11 & 0.076 & 0.076 & 0.116 & 0.130 & 0.126 & 0.093 & 0.134 & 0.203 & 0.125 & 0.389 & 0.131 & 0.007\end{array}$ $\begin{array}{llllllllllllll}11 & 0.188 & 0.189 & 0.173 & 0.192 & 0.176 & 0.198 & 0.199 & 0.200 & 0.172 & 0.334 & 0.179 & 0.191\end{array}$

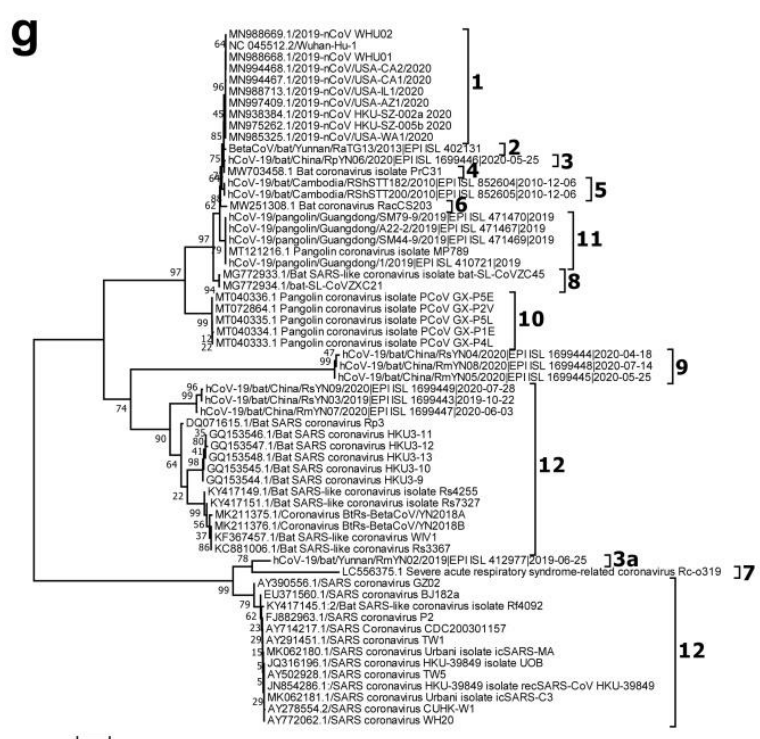

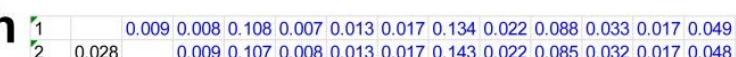

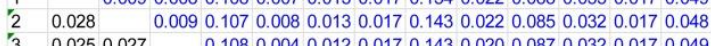 $\begin{array}{llllllllllllll}3 & 0.025 & 0.027 & 0.108 & 0.004 & 0.012 & 0.017 & 0.143 & 0.020 & 0.087 & 0.032 & 0.017 & 0.049\end{array}$ 3a $0.9640 .9480 .948 \quad 0.1090 .1020 .1110 .0390 .1000 .1770 .1090 .1140 .050$ $\begin{array}{llllllllllllll}4 & 0.018 & 0.021 & 0.006 & 0.960 & 0.011 & 0.017 & 0.141 & 0.021 & 0.088 & 0.032 & 0.016 & 0.049\end{array}$ $5 \quad 0.0510 .0540 .0440 .9030 .037 \quad 0.0170 .1350 .0230 .0830 .0350 .0190 .047$ $\begin{array}{lllllllllllll}6 & 0.090 & 0.086 & 0.086 & 0.953 & 0.083 & 0.084 & 0.167 & 0.022 & 0.088 & 0.034 & 0.017 & 0.049\end{array}$ $7 \quad 1.1061 .1501 .1630 .3281 .1481 .0981 .232 \quad 0.1340 .2140 .1370 .1390 .065$

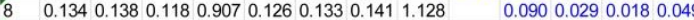 $9 \quad 0.7440 .7250 .7421 .2270 .7440 .7140 .7541 .3920 .774 \quad 0.0810 .0870 .073$ $\begin{array}{llllllllllllllll}10 & 0.251 & 0.246 & 0.250 & 0.936 & 0.250 & 0.265 & 0.259 & 1.088 & 0.218 & 0.707 & 0.030 & 0.045\end{array}$ $\begin{array}{lllllllllllllllll}11 & 0.088 & 0.090 & 0.084 & 0.985 & 0.080 & 0.106 & 0.093 & 1.140 & 0.107 & 0.742 & 0.243 & 0.047\end{array}$ 112 0.4050 .4020 .4070 .4100 .4130 .3820 .4030 .4900 .4070 .5520 .3910 .390

Extended Data Fig. 3

Extended Data Fig. 3: The genetic relationship of SARS-CoV-2 and related viruses in and net between-group mean distance respectively for SARS-CoV-2 related viruses. c \& d, 
The region 7 (22561-23161nt) specific phylogenetic tree and net between-group mean distance respectively for SARS-CoV-2 related viruses. e \& f , The region 8 (23221-27909nt) specific phylogenetic tree and net between-group mean distance respectively for SARS-CoV2 related viruses. $\mathbf{g} \boldsymbol{\&} \mathbf{h}$, The region 9 (27910 -28257nt) specific phylogenetic tree and net between-group mean distance respectively for SARS-CoV-2 related viruses.

a

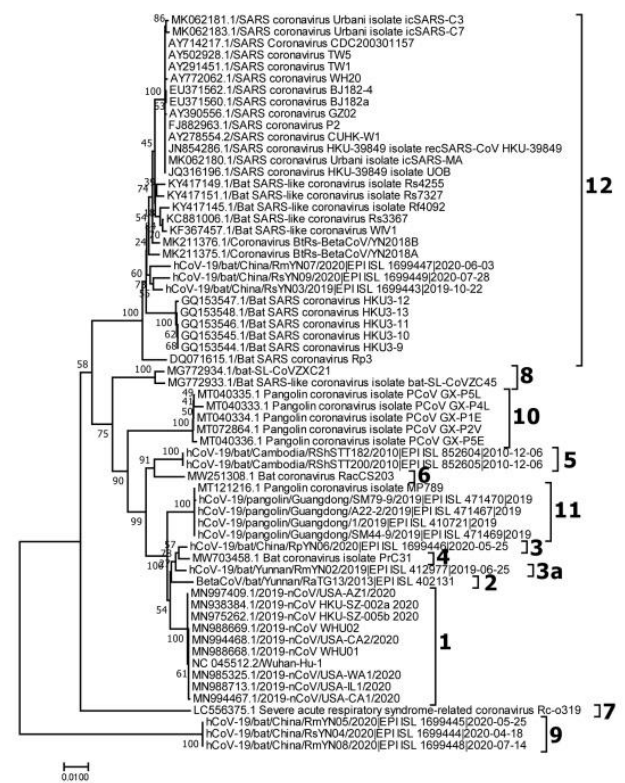

b

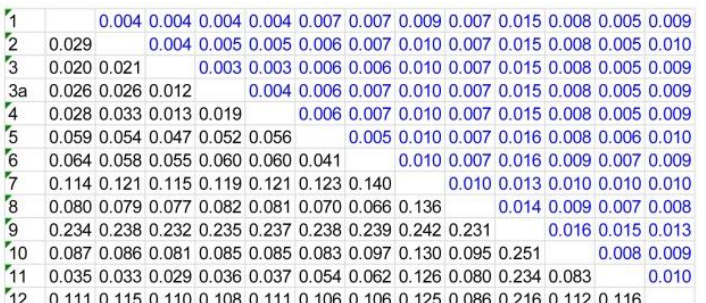

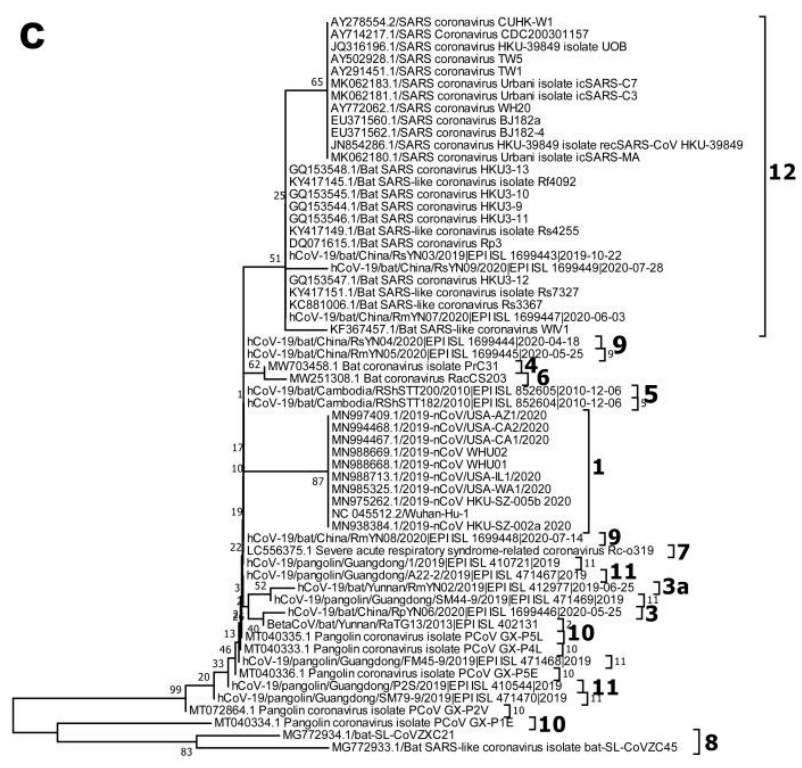

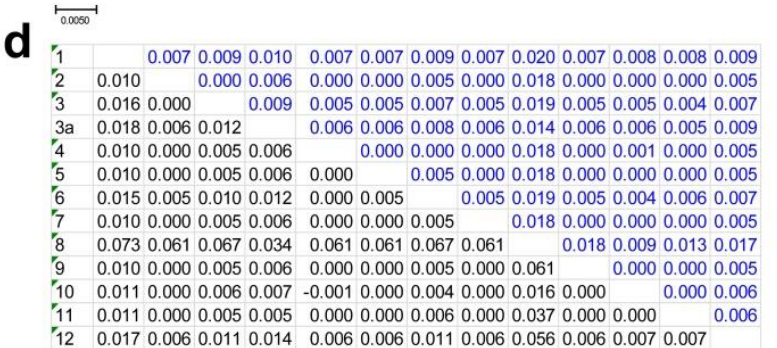
Extended Data Fig. 4

Extended Data Fig. 4: The genetic relationship of SARS-CoV-2 and related viruses in the region 10 and 11. a \& b, The region 10 (28258 -29674nt) specific phylogenetic tree and net between-group mean distance respectively for SARS-CoV-2 related viruses. c \& d, The region 11 (3'UTR) specific phylogenetic tree and net between-group mean distance respectively for SARS-CoV-2 related viruses. 


\begin{tabular}{|c|c|c|c|c|c|c|c|c|c|c|c|c|c|}
\hline 1 & & 0.006 & 0.020 & 0.039 & 0.042 & 0.061 & 0.059 & 0.060 & 0.059 & 0.059 & 0.056 & 0.080 & 0.081 \\
\hline 2 & 0.015 & & 0.019 & 0.039 & 0.042 & 0.060 & 0.058 & 0.060 & 0.058 & 0.059 & 0.055 & $0.0 / 9$ & 0.078 \\
\hline 3 & 0.121 & 0.115 & & 0.039 & 0.044 & 0.061 & 0.059 & 0.062 & 0.060 & 0.061 & 0.059 & 0.080 & 0.086 \\
\hline 4 & 0.379 & 0.375 & 0.384 & & 0.022 & 0.063 & 0.061 & 0.064 & 0.061 & 0.067 & 0.058 & 0.076 & 0.085 \\
\hline 5 & 0.404 & 0.400 & 0.426 & 0.151 & & 0.065 & 0.065 & & & & & & 0.085 \\
\hline 6 & 0.663 & 0.653 & 0.658 & 0.707 & 0.709 & & 0.013 & 0.0 & & 0.0 & & & 0.077 \\
\hline $6 a$ & 0.641 & 0.632 & 0.644 & 0.686 & 0.704 & 0.059 & & 0.034 & & & & & 0.073 \\
\hline $6 b$ & 0.68 & & & & & & 0.279 & & & & & 74 & 0.075 \\
\hline $6 c$ & 0.625 & 0.615 & 0.634 & 0.660 & 0.640 & 0.274 & 0.265 & 0.310 & & 0.037 & & & 0.074 \\
\hline $6 d$ & 0.643 & & & 0.725 & & 0.281 & 0.283 & 0.348 & 0.3 & & 0.045 & 0.0 & 0.070 \\
\hline 7 & 0.593 & 0.582 & 0.622 & 0.614 & 0.588 & 0.513 & 0.480 & 0.516 & 0.471 & 0.473 & & 0.060 & 0.063 \\
\hline $7 a$ & 0.885 & 0.88 & 0.868 & 0.85 & 0.86 & 0.803 & 0.822 & 0.822 & 0.854 & 0.772 & 0.656 & & 0.085 \\
\hline 8 & 0.929 & 0.906 & 0.968 & 0.972 & 0.922 & 0.880 & 0.847 & 0.835 & 0.834 & 0.780 & 0.730 & 0.989 & \\
\hline
\end{tabular}

b $\quad 7 \ldots \ldots$....172

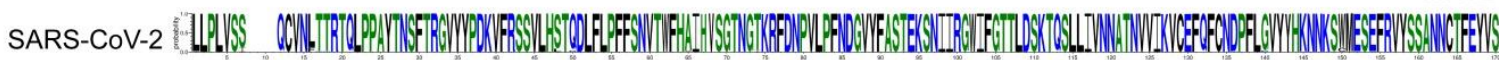

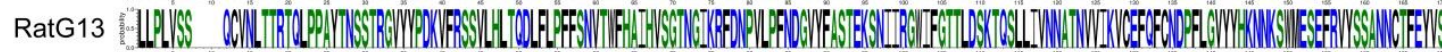

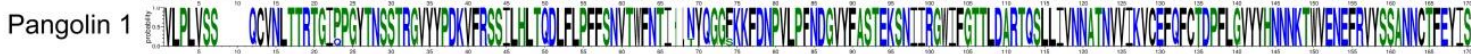

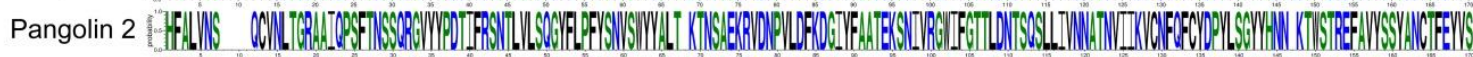

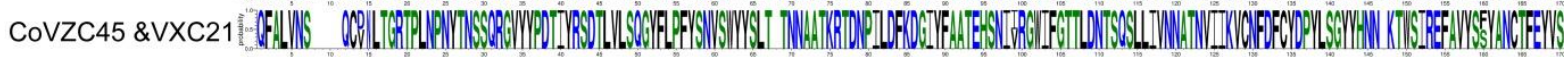

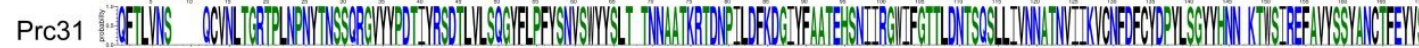

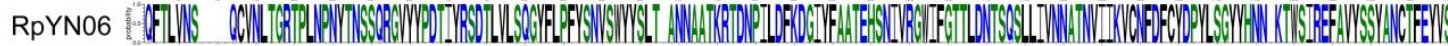

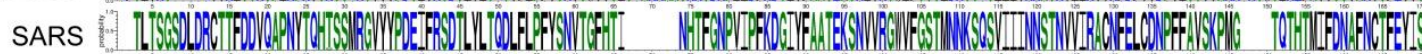

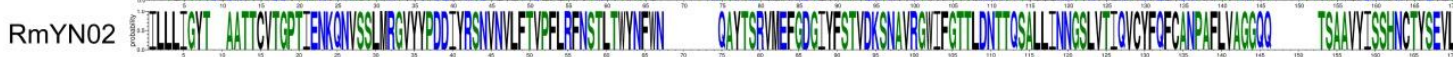

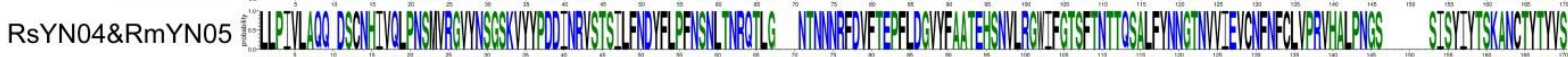

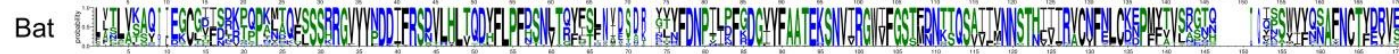
$173 \ldots$

$\ldots 332$

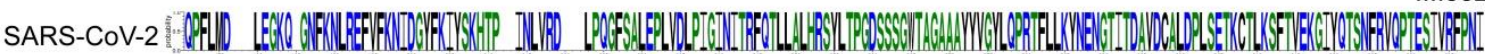

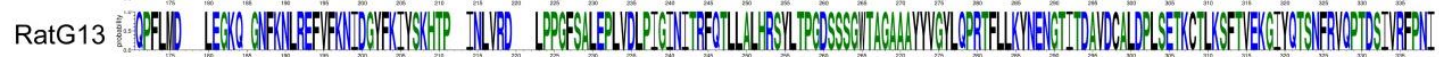

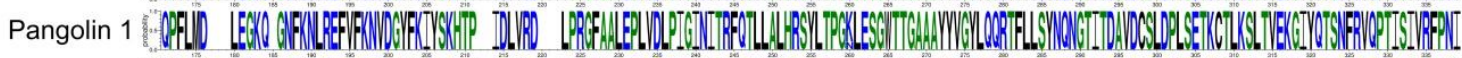

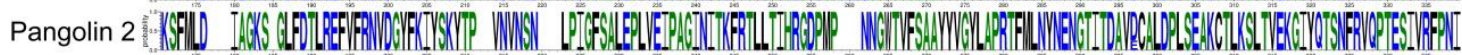

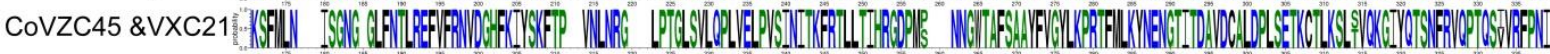

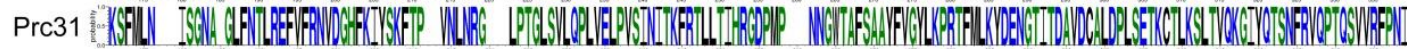

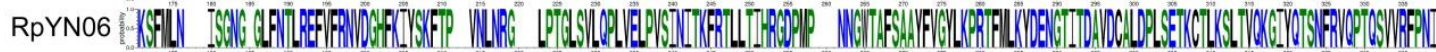

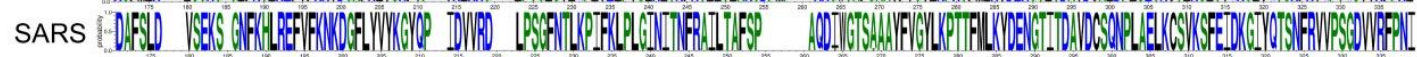

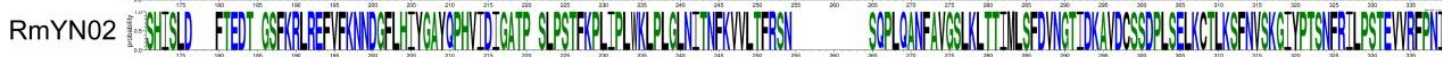

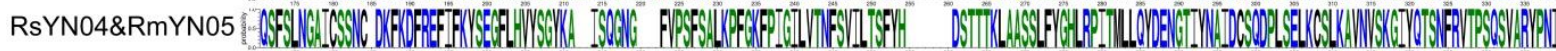

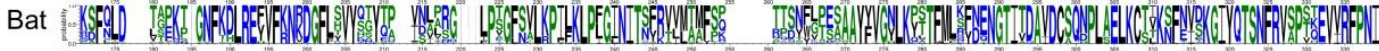

Extended Data Fig. 5

Extended Data Fig. 5: The Amino acid diversity in the S1-NTD (region 6). a, The region 6 amino acid-specific net between-group mean distance respectively for SARS-CoV-2 related viruses. b, The amino acid diversity in the different SARS viruses. 

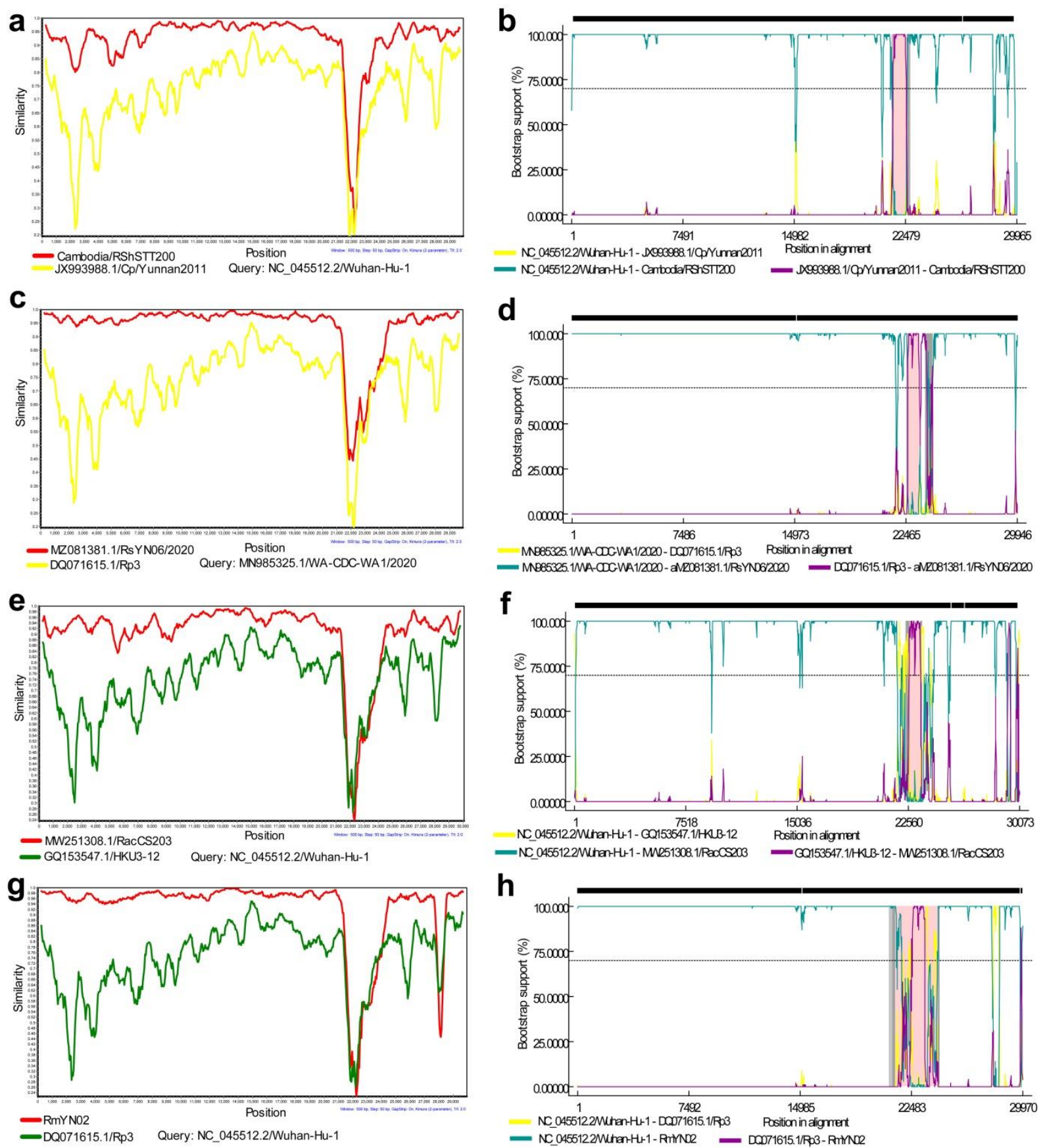

Extended Data Fig.6: Recombination in the clade-I SARS-CoV-2-rB-CoV. a \& b, The recombination analysis based on Similarity plot (a) and BOOTSCAN (b) using the complete genomes nucleotide sequences of the SARS-CoV-2, Cambodia bat virus RShSTT200 and Bat coronavirus $\mathrm{Cp} / \mathrm{Yunnan2011}$; SARS-CoV-2 used as a reference sequence. c \& d, The recombination analysis based on Similarity plot (c) and BOOTSCAN (d) using the complete genomes nucleotide sequences of the SARS-CoV-2, RsYN06, and Bat SARS coronavirus Rp3; SARS-CoV-2 used as a reference sequence. e \& f, The recombination analysis based on Similarity plot (e) and BOOTSCAN (f) using the complete genomes nucleotide sequences of the SARS-CoV-2, RacCS203, and Bat SARS coronavirus HKU3-12; SARS-CoV-2 used as a reference sequence. $\mathbf{g} \boldsymbol{\&} \mathbf{h}$, The recombination analysis based on Similarity plot ( $\mathrm{g}$ ) and BOOTSCAN (h) using the complete genomes nucleotide sequences of the SARS-CoV-2, RmYN02, and Bat SARS coronavirus Rp3; SARS-CoV-2 used as a reference sequence. 
a

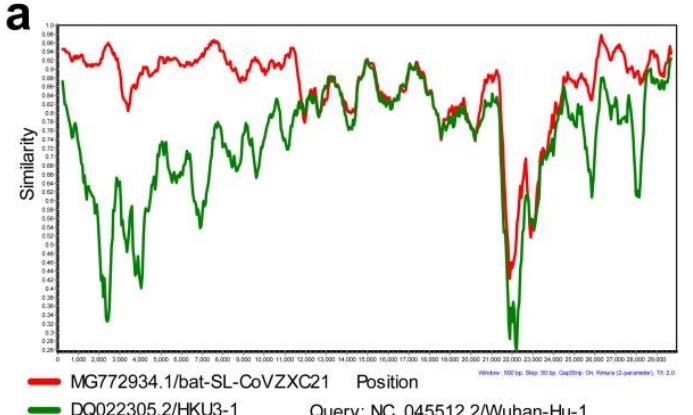

C

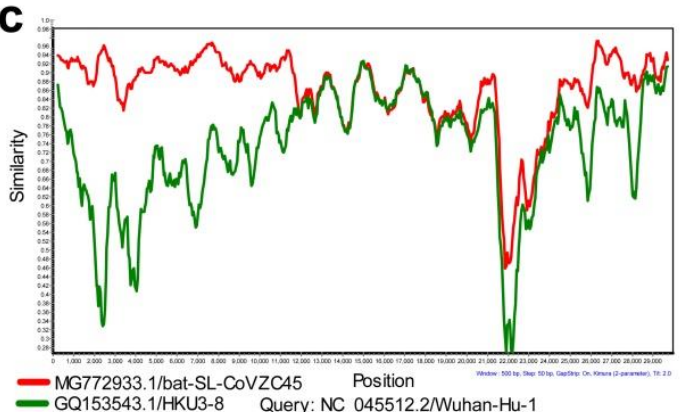

Extended Data Fig. 7

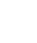
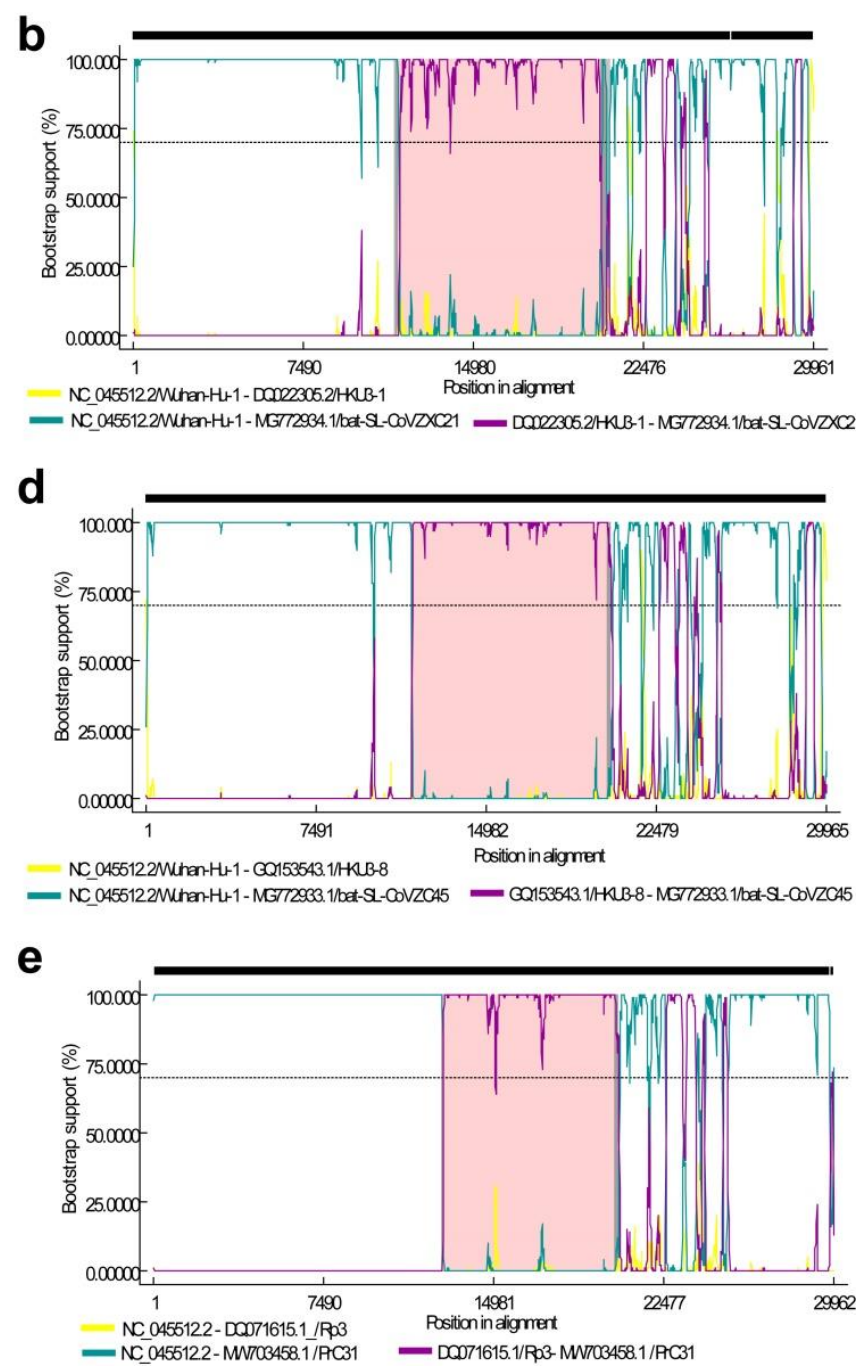

Extended Data Fig. 7: Recombination in the clade-II SARS-CoV-2-rB-CoV. a \& b, The recombination analysis based on Similarity plot (a) and BOOTSCAN (b) using the complete genomes nucleotide sequences of the SARS-CoV-2, bat-SL-CoVZXC21 and Bat SARS coronavirus HKU3-1; SARS-CoV-2 used as reference sequence. c \& d, The recombination analysis based on Similarity plot (c) and BOOTSCAN (d) using the complete genomes nucleotide sequences of the SARS-CoV-2, bat-SL-CoVZC45, and Bat SARS coronavirus HKU3-8; SARS-CoV-2 used as a reference sequence. e, The recombination analysis based on BOOTSCAN using the complete genomes nucleotide sequences of the SARS-CoV-2, bat coronavirus PrC31, and Bat SARS coronavirus Rp3. 
a
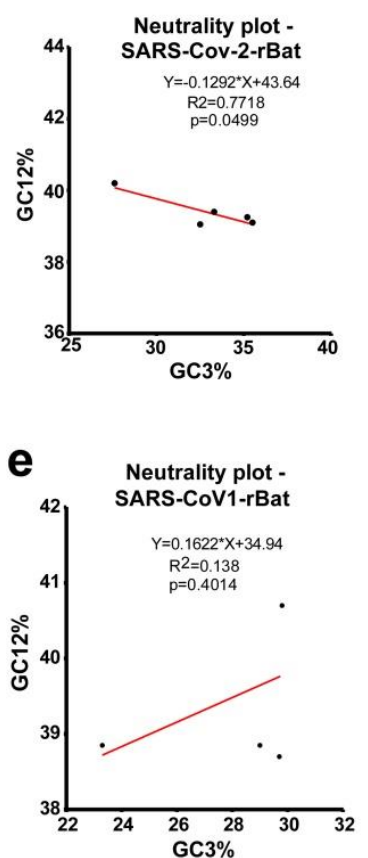

b

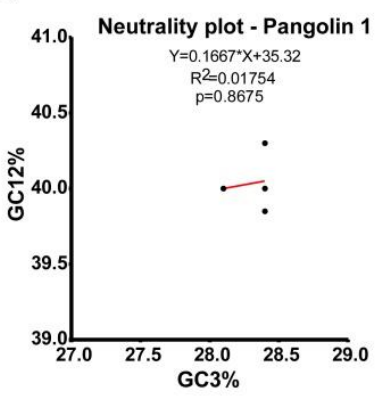

f

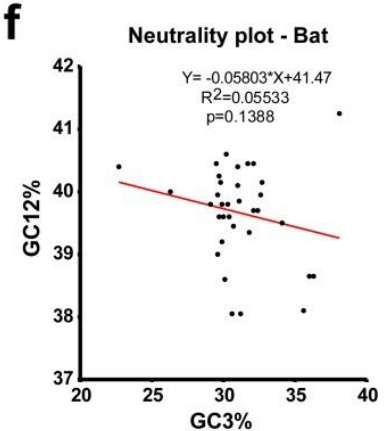

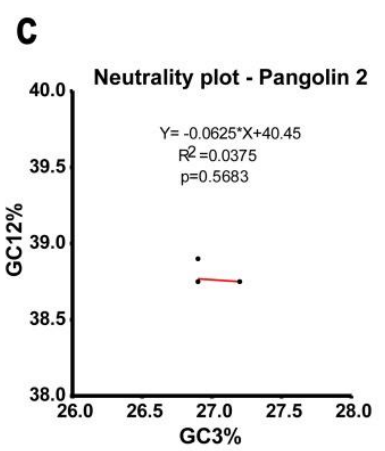
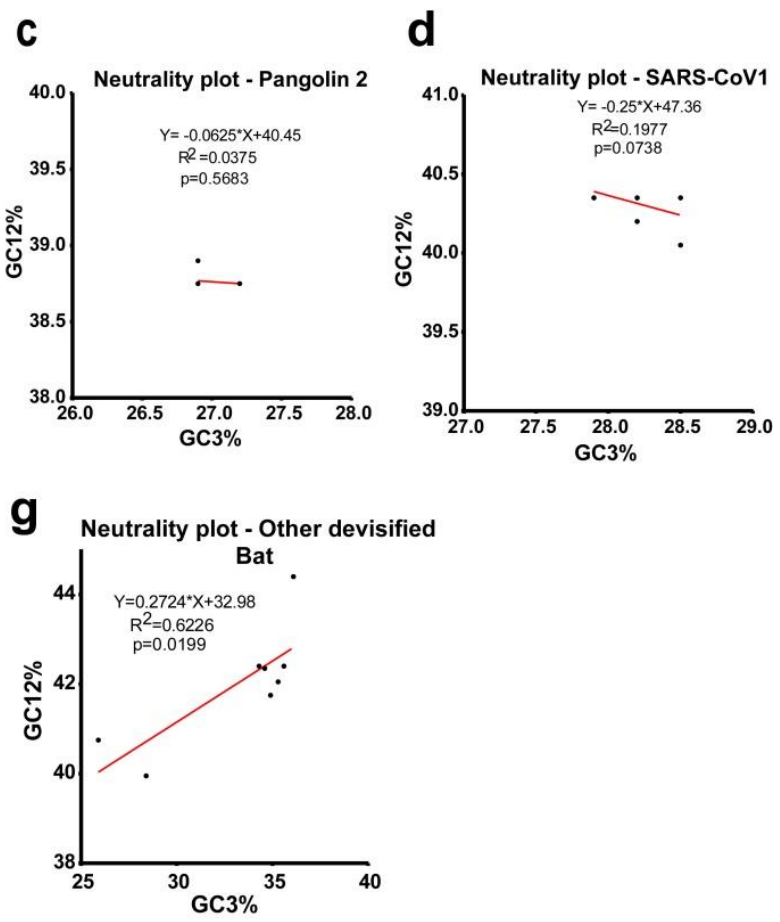

Extended Data Fig. 8

Extended Data Fig. 8: The Neutrality plot analysis in the S1-NTD (region 6). a, SARSCov-2-rBat; b, Pangolin/GX/2018 lineage; c, Pangolin/Guangdong/2019 lineage; d, SARSCoV-1; e, SARS-Cov-1-rBat; f, Bat-SARS viruses; g, Other diversified bat Bat-SARS viruses.

(1)

9

0

1

2

3

4


a

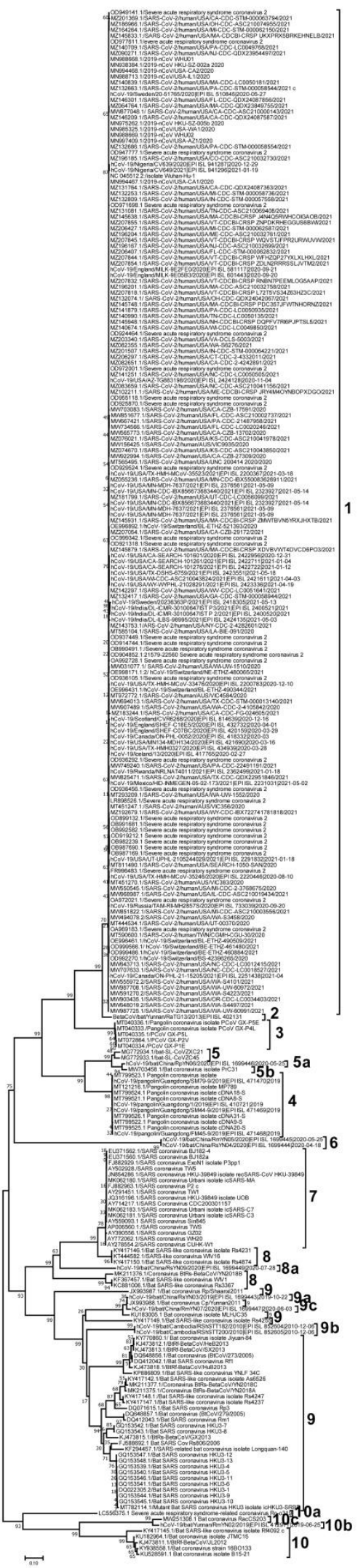

Supplementary Fig.1: Phylogenetic relationship S1-NTD (region 6). a, The region

1126 (21578-22560nt) specific phylogenetic tree for SARS-CoV-2 related viruses. b, The region 1136 specific amino acid-based phylogenetic tree for SARS-CoV-2 related viruses.

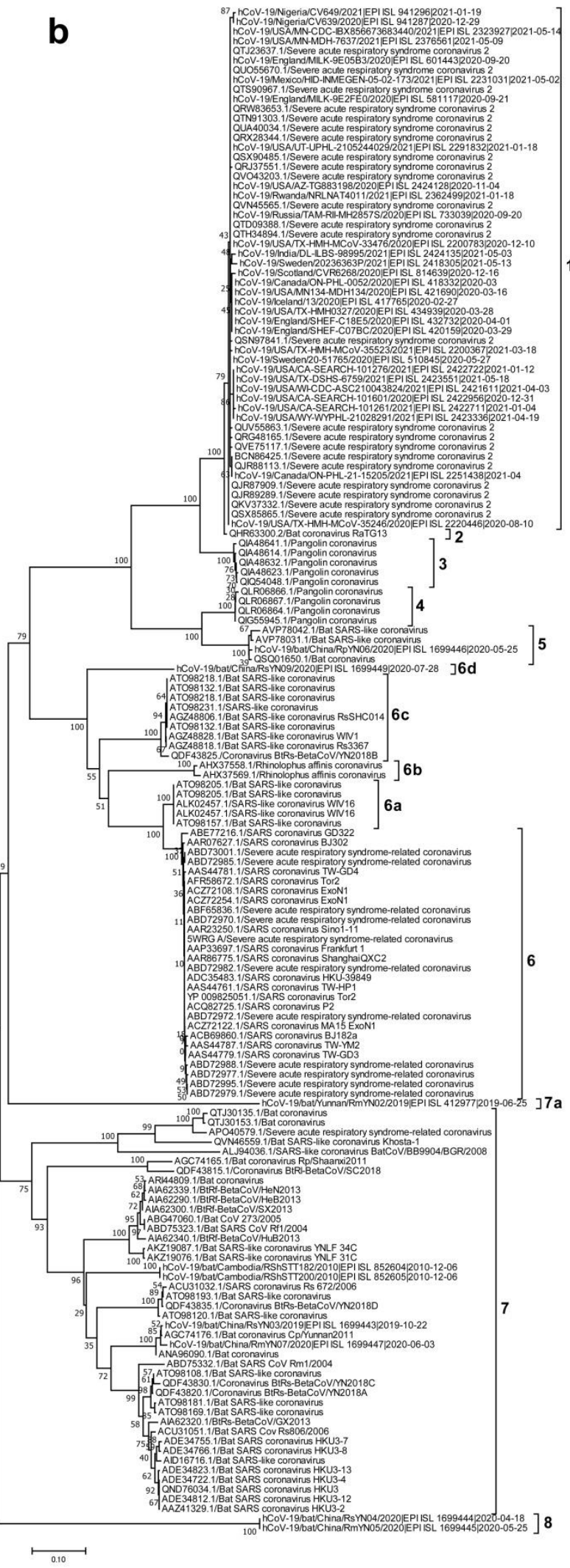

\section{Supplementary Figure 1}


a

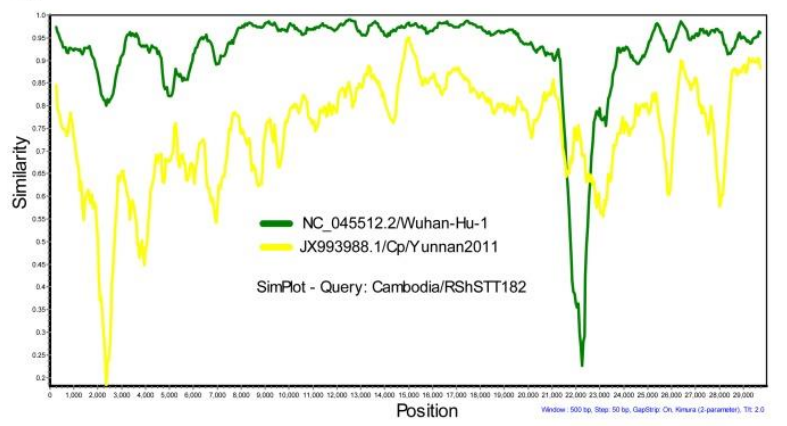

C

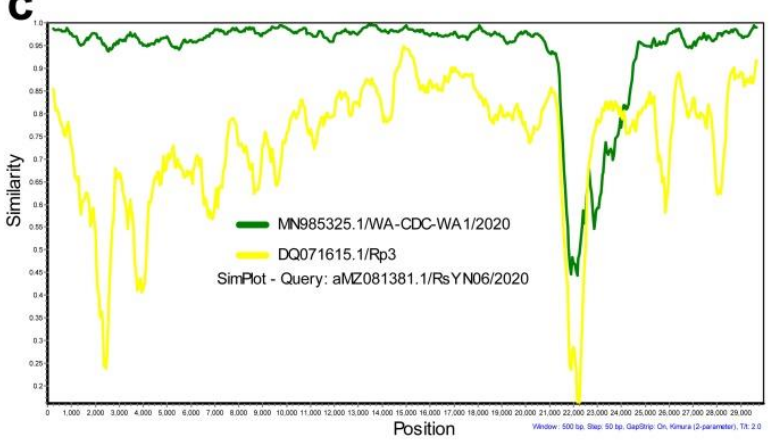

e

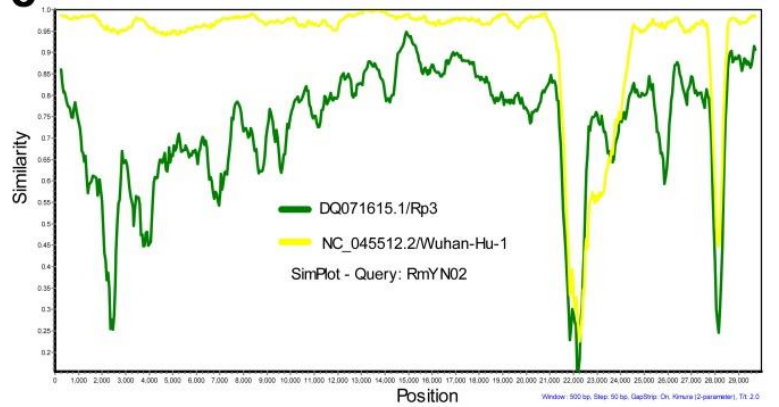

g

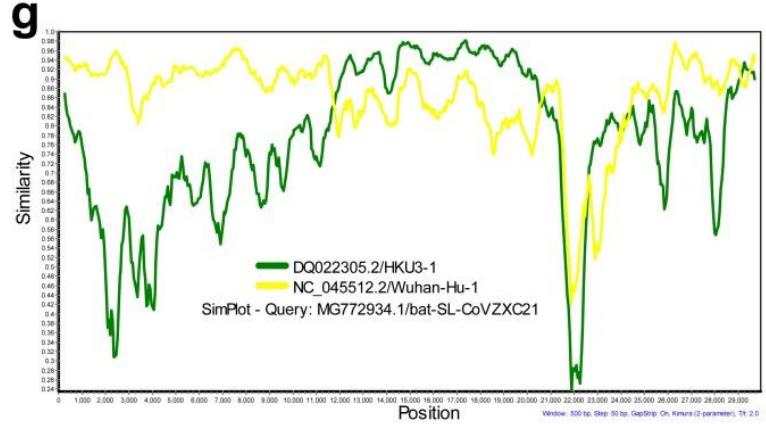

b

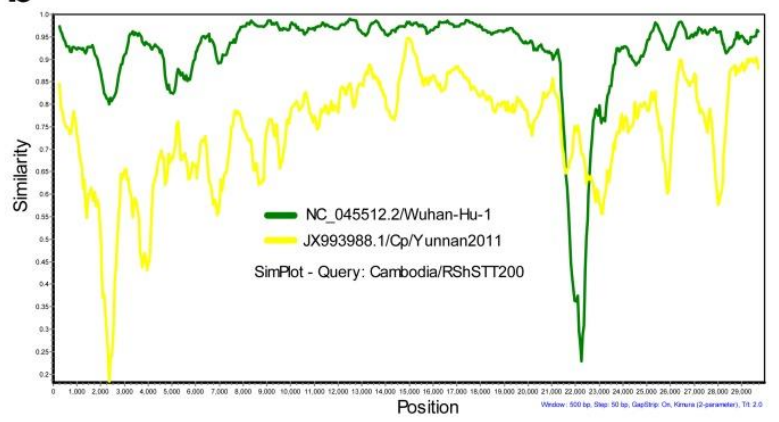

d

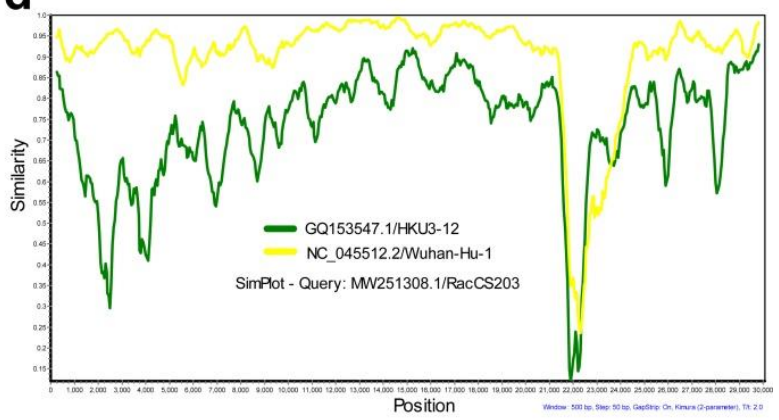

$\mathbf{f}$

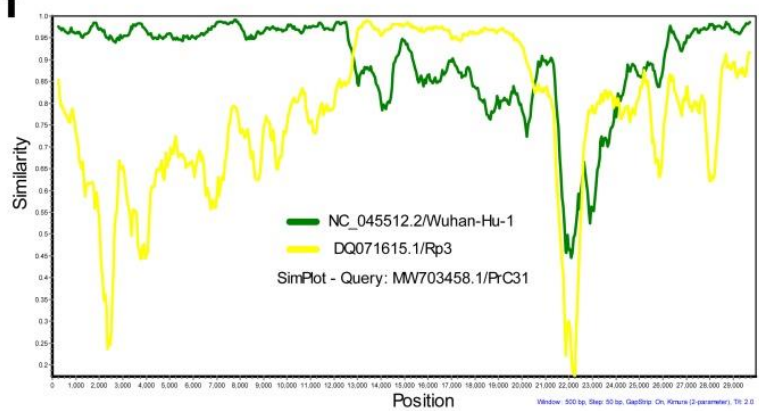

h

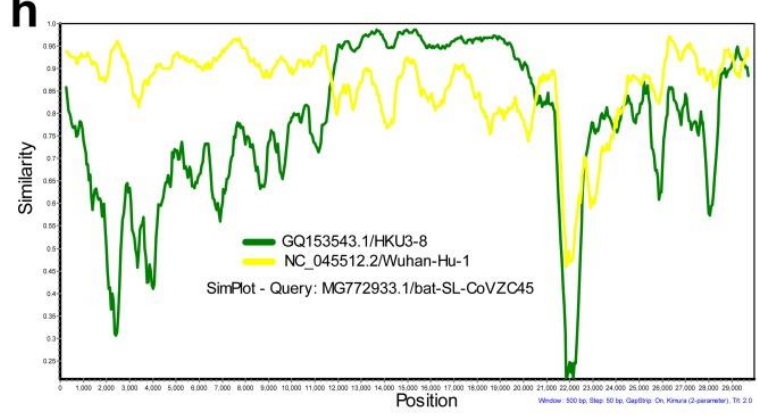

Supplementary Figure 2

Supplementary Fig.1: The Similarity plot analysis of SARS-CoV-2-rB-CoV. a, Similarity plot based on the complete genomes nucleotide sequences of the SARS-CoV-2, Cambodia bat virus RShSTT182 and Bat coronavirus Cp/Yunnan2011; Cambodia bat virus RShSTT182 used as a reference sequence. b, Similarity plot based on the complete genomes nucleotide sequences of the SARS-CoV-2, Cambodia bat virus RShSTT200 and Bat coronavirus Cp/Yunnan2011; Cambodia bat virus RShSTT200 used as a reference sequence. c, Similarity plot based on the complete genomes nucleotide sequences of the SARS-CoV-2, RsYN06, and Bat SARS coronavirus Rp3; RsYN06 used as a reference sequence. d, Similarity plot based on the complete genomes nucleotide sequences of the SARS-CoV-2, RacCS203, and Bat SARS coronavirus HKU3-12; RacCS203 used as a reference sequence. e, 
130 RmYN02, and Bat SARS coronavirus Rp3; RmYN02 used as a reference sequence. f, 131 Similarity plot based on the complete genomes nucleotide sequences of the SARS-CoV-2, bat 132 coronavirus PrC31, and Bat SARS coronavirus Rp3; bat coronavirus PrC31 used as a 133 reference sequence. $\mathbf{g}$, Similarity plot based on the complete genomes nucleotide sequences 134 of the SARS-CoV-2, bat-SL-CoVZXC21, and Bat SARS coronavirus HKU3-1; bat-SL135 CoVZXC21 used as a reference sequence. $\mathbf{h}$, Similarity plot based on the complete genomes 136 nucleotide sequences of the SARS-CoV-2, bat-SL-CoVZC45, and Bat SARS coronavirus 137 HKU3-8; bat-SL-CoVZC45 used as a reference sequence. 